\title{
Central Extensions of Root Graded Lie Algebras
}

\author{
by
}

\author{
Malihe YousofzADEH
}

\begin{abstract}
We study central extensions of Lie algebras graded by an irreducible locally finite root system.
\end{abstract}

2010 Mathematics Subject Classification: Primary 17B70; Secondary 17B60.

Keywords: root graded Lie algebras, central extensions.

\section{§1. Introduction}

Motivated by work of G. Seligman [13], S. Berman and V. Moody [8] introduced the notion of a root graded Lie algebra. They defined a Lie algebra graded by an irreducible reduced finite root system. Their definition was extended to Lie algebras graded by a reduced locally finite root system by E. Neher [10] and also to Lie algebras graded by an irreducible finite root system of type $B C$ by B. Allison, G. Benkart and Y. Gao [3] in 2002. Finally, this definition was generalized to Lie algebras graded by a locally finite root system in [11] and [19]. Root graded Lie algebras play an important role in infinite-dimensional Lie theory, namely, they are important in the study of extended affine Lie algebras [1]. More precisely, the so-called centerless core of an extended affine Lie algebra $\mathcal{L}$, which is the main part of the structure of $\mathcal{L}$, is a specific root graded Lie algebra called a Lie torus; see [4], [17] and [11].

A complete description of Lie algebras graded by an irreducible finite root system is given in [8], [7], [3] and [6]. Also in [10], the author realizes Lie algebras graded by an irreducible reduced locally finite root system which is not of type $F_{4}, G_{2}$ or $E_{8}$, as central extensions of Tits-Kantor-Koecher algebras of certain Jordan pairs.

Communicated by H. Nakajima. Received April 3, 2012. Revised October 4, 2012, and February $1,2013$.

M. Yousofzadeh: Department of Mathematics, University of Isfahan, P.O. Box 81745-163, Isfahan, Iran, and School of Mathematics, Institute for Research in Fundamental Sciences (IPM), P.O. Box 19395-5746, Tehran, Iran;

e-mail: ma.yousofzadeh@sci.ui.ac.ir 
Because of the important role of central extensions in the theory of Lie algebras, the next natural step in the study of root graded Lie algebras is to consider their central extensions. Central extensions of Lie algebras graded by an irreducible finite root system are studied in [2], [3] and [6]. Using the description of the universal central extension of a root graded Lie algebra of finite type as in [2], the authors of [18] and [5] find a finite presentation for the universal central extension of a Lie torus of finite type $X \neq A, C, B C$. A. Welte [15], in her Ph.D. thesis, studies central extensions of a root graded Lie algebra of reduced type using Tits-Kantor-Koecher structure and describes the universal central extension of Lie algebras graded by locally finite root systems of type $A$ and $C$. In [12], E. Neher and J. Sun prove that the universal central extension of a direct limit of a class $\left\{\mathcal{L}_{i} \mid i \in I\right\}$ of perfect Lie superalgebras coincides with the direct limit of the universal central extensions of the $\mathcal{L}_{i}$ 's. Using this together with the fact that the center of a centrally closed Lie algebra graded by an irreducible reduced finite root system $R$ with coordinate algebra $\mathfrak{a}$ just depends on $\mathfrak{a}$ and the type of $R$ and is independent of the rank of $R$, they determine the universal central extension of certain direct limits of Lie algebras graded by irreducible reduced finite root systems; see [12, Example 2.10] as well as [3], [2].

In [19], the author studies Lie algebras graded by an infinite irreducible locally finite root system which is not necessarily reduced, and gives a complete description of these Lie algebras. The most important ingredient in this description is a quadruple called a coordinate quadruple. In this paper, we study central extensions of root graded Lie algebras. We prove that for an irreducible locally finite root system $R$, a perfect central extension of an $R$-graded Lie algebra with associated coordinate quadruple $\mathfrak{q}$ is an $R$-graded Lie algebra with the same coordinate quadruple. We give a complete description of perfect central extensions of a root graded Lie algebra $\mathcal{L}$ in terms of the above ingredient involved in the description of $\mathcal{L}$. In particular, we completely determine the universal central extension of a Lie algebra graded by an irreducible locally finite root system. We hope our description of the universal central extension of a root graded Lie algebra offers a nice presentation for the universal central extension of a Lie torus.

\section{$\S 2$. Preliminaries}

Throughout this work, $\mathbb{N}$ denotes the set of nonnegative integers and $\mathbb{F}$ is a field of characteristic zero. Unless otherwise mentioned, all vector spaces are considered over $\mathbb{F}$. For a vector space $V$, we denote by $V^{*}$ the dual space of $V$ and use $\operatorname{End}_{\mathbb{F}}(V)$ (or $\operatorname{End}(V)$ if there is no confusion) to denote the vector space of all linear endomorphisms of $V$. For a linear transformation $T \in \operatorname{End}_{\mathbb{F}}(V)$, if the trace 
of $T$ is defined, we denote it by $\operatorname{tr}(T)$. Also for a nonempty set $S$, by $\operatorname{id}_{S}$ we mean the identity map on $S$, and by $|S|$ the cardinality of $S$. For a nonempty index set $I$, by notational convention, we take $\bar{I}:=\{\bar{i} \mid i \in I\}$ to be a disjoint copy of $I$, and for each subset $J$ of $I$, by $\bar{J}$ we mean the subset of $\bar{I}$ corresponding to $J$.

Definition $2.1([9, \S 3])$. Let $\mathcal{U}$ be a nontrivial vector space and $R$ be a subset of $\mathcal{U}$. The subset $R$ is said to be a locally finite root system in $\mathcal{U}$ of $\operatorname{rank} \operatorname{dim}(\mathcal{U})$ if the following conditions are satisfied:

(i) $R$ is locally finite, contains zero and spans $\mathcal{U}$.

(ii) For every $\alpha \in R^{\times}:=R \backslash\{0\}$, there exists $\check{\alpha} \in \mathcal{U}^{*}$ such that $\check{\alpha}(\alpha)=2$ and $s_{\alpha}(\beta) \in R$ for $\alpha, \beta \in R$, where $s_{\alpha}: \mathcal{U} \rightarrow \mathcal{U}$ maps $u \in \mathcal{U}$ to $u-\check{\alpha}(u) \alpha$.

(iii) $\check{\alpha}(\beta) \in \mathbb{Z}$ for $\alpha, \beta \in R^{\times}$.

Set $R_{\text {sdiv }}:=(R \backslash\{\alpha \in R \mid 2 \alpha \in R\}) \cup\{0\}$ and call it the semi-divisible subsystem of $R$. The root system $R$ is called reduced if $R=R_{\text {sdiv }}$.

Suppose that $R$ is a locally finite root system. A nonempty subset $S$ of $R$ is said to be a subsystem of $R$ if $S$ contains zero and $s_{\alpha}(\beta) \in S$ for $\alpha, \beta \in S \backslash\{0\}$. A subsystem $S$ of $R$ is called full if $\left(\operatorname{span}_{\mathbb{F}} S\right) \cap R=S$. Following [9, §3.12], we say nonzero roots $\alpha, \beta$ of a subset $S$ of $R$ are connected in $S$ if there exist finitely many nonzero roots $\alpha_{1}:=\alpha, \alpha_{2}, \ldots, \alpha_{n}:=\beta$ in $S$ such that $\check{\alpha}_{i+1}\left(\alpha_{i}\right) \neq 0,1 \leq i \leq n-1$. Connectedness in $R^{\times}$defines an equivalence relation in $R^{\times}$and its equivalence are classes are called connected components of $R$. A nonempty subset $X$ of $R$ is called irreducible if any two nonzero elements $x, y \in X$ are connected in $X$.

Two locally finite root systems $(R, \mathcal{U})$ and $(S, \mathcal{V})$ are said to be isomorphic if there is a linear transformation $f: \mathcal{U} \rightarrow \mathcal{V}$ such that $f(R)=S$.

Suppose that $I$ is a nonempty index set and $\mathcal{U}:=\bigoplus_{i \in I} \mathbb{F} \epsilon_{i}$ is the free $\mathbb{F}$-module over the set $I$. Define the form

$$
(\cdot, \cdot): \mathcal{U} \times \mathcal{U} \rightarrow \mathbb{F}, \quad\left(\epsilon_{i}, \epsilon_{j}\right)=\delta_{i, j} \quad \text { for } i, j \in I,
$$

and set

$$
\begin{aligned}
\dot{A}_{I} & :=\left\{\epsilon_{i}-\epsilon_{j} \mid i, j \in I\right\}, \\
D_{I} & :=\dot{A}_{I} \cup\left\{ \pm\left(\epsilon_{i}+\epsilon_{j}\right) \mid i, j \in I, i \neq j\right\}, \\
B_{I} & :=D_{I} \cup\left\{ \pm \epsilon_{i} \mid i \in I\right\}, \\
C_{I} & :=D_{I} \cup\left\{ \pm 2 \epsilon_{i} \mid i \in I\right\}, \\
B C_{I} & :=B_{I} \cup C_{I} .
\end{aligned}
$$

One can see that these are irreducible locally finite root systems in their $\mathbb{F}$-spans which we refer to as type $A, D, B, C$ and $B C$ respectively. Moreover every irre- 
ducible locally finite root system of infinite rank is isomorphic to one of these root systems (see $[9, \S 4.14, \S 8]$ ). We note that $D_{I}, B_{I}, C_{I}$ and $B C_{I}$ span $\mathcal{U}$, while $\dot{A}_{I}$ spans a subspace of $\mathcal{U}$ of codimension one; for this reason, $\dot{A}_{I}$ is used instead of $A_{I}$ in the literature. Now we suppose $R$ is an irreducible locally finite root system as above and note that $(\alpha, \alpha) \in \mathbb{N}$ for all $\alpha \in R$. This allows us to define

$$
\begin{aligned}
& R_{\mathrm{sh}}:=\left\{\alpha \in R^{\times} \mid(\alpha, \alpha) \leq(\beta, \beta) \text { for all } \beta \in R\right\}, \\
& R_{\mathrm{ex}}:=R \cap 2 R_{\mathrm{sh}}, \\
& R_{\mathrm{lg}}:=R^{\times} \backslash\left(R_{\mathrm{sh}} \cup R_{\mathrm{ex}}\right) .
\end{aligned}
$$

The elements of $R_{\mathrm{sh}}$ (resp. $R_{\mathrm{lg}}, R_{\mathrm{ex}}$ ) are called short roots (resp. long roots, extralong roots) of $R$.

Definition 2.2. Let $\mathcal{H}$ be a Lie algebra. We say an $\mathcal{H}$-module $\mathcal{M}$ has a weight space decomposition with respect to $\mathcal{H}$ if

$$
\mathcal{M}=\bigoplus_{\alpha \in \mathcal{H}^{*}} \mathcal{M}_{\alpha} \quad \text { where } \quad \mathcal{M}_{\alpha}:=\{x \in \mathcal{M} \mid h \cdot x=\alpha(h) x, \forall h \in \mathcal{H}\}
$$

for all $\alpha \in \mathcal{H}^{*}$. The set $R:=\left\{\alpha \in \mathcal{H}^{*} \mid \mathcal{M}_{\alpha} \neq\{0\}\right\}$ is called the set of weights of $\mathcal{M}$ (with respect to $\mathcal{H}$ ). For $\alpha \in R, \mathcal{M}_{\alpha}$ is called a weight space, and any element of $\mathcal{M}_{\alpha}$ is called a weight vector of weight $\alpha$. If a Lie algebra $\mathcal{L}$ has a weight space decomposition with respect to a nontrivial subalgebra $H$ of $\mathcal{L}$ via the adjoint representation, $H$ is called a split toral subalgebra. The set of weights of $\mathcal{L}$ is called the root system of $\mathcal{L}$ with respect to $H$, and the corresponding weight spaces are called the root spaces of $\mathcal{L}$. A Lie algebra $\mathcal{L}$ is called split if it contains a splitting Cartan subalgebra, that is, a split toral subalgebra $H$ of $\mathcal{L}$ with $\mathcal{L}_{0}=H$. A locally finite split simple Lie algebra is said to be of type $A, B, C$ or $D$ if its corresponding root system with respect to a splitting Cartan subalgebra is of type $A, B, C$ or $D$ respectively.

Suppose that $J$ is a nonempty index set and $\mathcal{V}:=\mathcal{V}_{J}(\mathbb{F})\left(\right.$ or $\mathcal{V}_{J}$ if there is no confusion) is a vector space with a fixed basis $\left\{v_{j} \mid j \in J\right\}$. Then $\mathfrak{g l}_{\mathbb{F}}(\mathcal{V}):=\operatorname{End}(\mathcal{V})$ together with the commutator product is a Lie algebra. Now for $j, k \in J$, define

$$
e_{j, k}: \mathcal{V} \rightarrow \mathcal{V}, \quad v_{i} \mapsto \delta_{k, i} v_{j} \quad(i \in J) ;
$$

then $\mathfrak{g l}_{\mathbb{F}}(J):=\operatorname{span}_{\mathbb{F}}\left\{e_{j, k} \mid j, k \in J\right\}$ is a Lie subalgebra of $\mathfrak{g l}_{\mathbb{F}}(\mathcal{V})$.

Classical Lie algebra of type A: Suppose that $I$ is a nonempty index set of cardinality greater than 1 , and $\mathcal{V}$ is a vector space with a basis $\left\{v_{i} \mid i \in I\right\}$. Then

$$
\mathfrak{s l}(I):=\left\{\phi \in \mathfrak{g l}_{\mathbb{F}}(I) \mid \operatorname{tr}(\phi)=0\right\}
$$


is a locally finite split simple Lie subalgebra of $\mathfrak{g l}_{\mathbb{F}}(I)$ with splitting Cartan subalgebra

$$
\mathcal{H}:=\operatorname{span}_{\mathbb{F}}\left\{e_{i, i}-e_{j, j} \mid i, j \in I\right\}
$$

and corresponding root system isomorphic to $\dot{A}_{I}$.

Classical Lie algebra of type B: Suppose that $I$ is a nonempty index set. Take $J:=\{0\} \uplus I \uplus \bar{I}$ and consider the vector space $\mathcal{V}:=\mathcal{V}_{J}$ as above. Define the symmetric bilinear form $(\cdot, \cdot)$ on $\mathcal{V}$ by

$$
\begin{aligned}
& \left(v_{\bar{k}}, v_{j}\right):=2 \delta_{j, k}, \quad\left(v_{0}, v_{0}\right):=2, \\
& \left(v_{j}, v_{k}\right):=\left(v_{j}, v_{0}\right):=\left(v_{\bar{j}}, v_{0}\right):=\left(v_{\bar{j}}, v_{\bar{k}}\right):=0
\end{aligned}
$$

for $j, k \in I$. Then

$$
\mathfrak{o}_{B}(I):=\left\{\phi \in \mathfrak{g l}_{\mathbb{F}}(J) \mid(\phi(v), w)=-(v, \phi(w)) \text { for all } v, w \in \mathcal{V}\right\}
$$

is a locally finite split simple Lie subalgebra of $\mathfrak{g l}_{\mathbb{F}}(J)$ with splitting Cartan subalgebra

$$
\mathcal{H}:=\operatorname{span}_{\mathbb{F}}\left\{h_{i}:=e_{i, i}-e_{\bar{i}, \bar{i}} \mid i \in I\right\}
$$

and corresponding root system isomorphic to $B_{I}$.

Classical Lie algebra of type $C$ : Suppose that $I$ is a nonempty index set and $J:=I \uplus \bar{I}$. Consider the bilinear form $(\cdot, \cdot)$ on $\mathcal{V}:=\mathcal{V}_{J}$ defined by

$$
\left(v_{j}, v_{\bar{k}}\right):=-\left(v_{\bar{k}}, v_{j}\right):=2 \delta_{j, k}, \quad\left(v_{j}, v_{k}\right):=0, \quad\left(v_{\bar{j}}, v_{\bar{k}}\right):=0 \quad(j, k \in I) .
$$

Then

$$
\left(\mathfrak{s p}_{\mathbb{F}}(I):=\right) \mathfrak{s p}(I):=\left\{\phi \in \mathfrak{g l}_{\mathbb{F}}(J) \mid(\phi(v), w)=-(v, \phi(w)) \text { for all } v, w \in \mathcal{V}\right\}
$$

is a locally finite split simple Lie subalgebra of $\mathfrak{g l}_{\mathbb{F}}(J)$ with splitting Cartan subalgebra

$$
\mathcal{H}:=\operatorname{span}_{\mathbb{F}}\left\{h_{i}:=e_{i, i}-e_{\bar{i}, \bar{i}} \mid i \in I\right\}
$$

and corresponding root system isomorphic to $C_{I}$.

Moreover if we define

$$
\rho_{1}: \mathfrak{s p}(I) \rightarrow \operatorname{End}(\mathcal{V}), \quad \rho_{1}(\phi)(v):=\phi(v), \quad \phi \in \mathfrak{s p}(I), v \in \mathcal{V},
$$

then $\rho_{1}$ is an irreducible representation of $\mathfrak{s p}(I)$ in $\mathcal{V}$ equipped with a weight space decomposition with respect to $\mathcal{H}$ whose set of weights is $\left\{ \pm \epsilon_{i} \mid i \in I\right\}$ with 
$\mathcal{V}_{\epsilon_{i}}=\mathbb{F} v_{i}$ and $\mathcal{V}_{-\epsilon_{i}}=\mathbb{F} v_{i}$ for $i \in I$. Also for

$$
\mathcal{S}:=\left\{\phi \in \mathfrak{g l}_{\mathbb{F}}(J) \mid \operatorname{tr}(\phi)=0,(\phi(v), w)=(v, \phi(w)) \text { for all } v, w \in \mathcal{V}\right\},
$$

the map

$$
\rho_{2}: \mathfrak{s p}(I) \rightarrow \operatorname{End}(\mathcal{S}), \quad \rho_{2}(X)(Y):=[X, Y], \quad X \in \mathfrak{s p}(I), Y \in \mathcal{S},
$$

defines an irreducible representation of $\mathfrak{s p}(I)$ in $\mathcal{S}$ equipped with a weight space decomposition with respect to $\mathcal{H}$ whose set of weights is $\left\{0, \pm\left(\epsilon_{i} \pm \epsilon_{j}\right) \mid i, j \in I\right.$, $i \neq j\}$.

Classical Lie algebra of type $D$ : Suppose that $I$ is a nonempty index set and $J:=I \uplus \bar{I}$. Define the symmetric bilinear form $(\cdot, \cdot)$ on $\mathcal{V}:=\mathcal{V}_{J}$ by

$$
\left(v_{\bar{k}}, v_{j}\right):=2 \delta_{j, k}, \quad\left(v_{j}, v_{k}\right):=\left(v_{\bar{j}}, v_{\bar{k}}\right):=0 \quad(j, k \in I) .
$$

Then

$$
\mathfrak{o}_{D}(I):=\left\{\phi \in \mathfrak{g l}_{\mathbb{F}}(J) \mid(\phi(v), w)=-(v, \phi(w)) \text { for all } v, w \in \mathcal{V}\right\}
$$

is a locally finite split simple Lie subalgebra of $\mathfrak{g l}_{\mathbb{F}}(J)$ with splitting Cartan subalgebra

$$
\mathcal{H}:=\operatorname{span}_{\mathbb{F}}\left\{h_{i}:=e_{i, i}-e_{\bar{i}, \bar{i}} \mid i \in I\right\}
$$

and corresponding root system isomorphic to $D_{I}$.

Lemma 2.3 ([12, Thm. VI.7]). Suppose that I is an infinite index set. Then $\mathfrak{o}_{B}(I)$ is isomorphic to $\mathfrak{o}_{D}(I)$. Moreover, if $\mathcal{G}$ is an infinite-dimensional locally finite split simple Lie algebra, then $\mathcal{G}$ is isomorphic to exactly one of the Lie algebras $\mathfrak{s l}(I)$, $\mathfrak{o}_{B}(I)$ or $\mathfrak{s p}(I)$, for some infinite index set $I$.

Definition 2.4. Suppose that $R$ is an irreducible locally finite root system. We say a Lie algebra $\mathcal{L}$ is $R$-graded or (graded by $R$ ) with grading pair $(\mathcal{G}, \mathcal{H})$ if the following conditions are satisfied:

(i) $\mathcal{G}$ is a locally finite split simple Lie subalgebra of $\mathcal{L}$ with splitting Cartan subalgebra $\mathcal{H}$ and corresponding root system $R_{\text {sdiv }}$.

(ii) $\mathcal{L}$ has a weight space decomposition $\mathcal{L}=\bigoplus_{\alpha \in R} \mathcal{L}_{\alpha}$ with respect to $\mathcal{H}$ via the adjoint representation.

(iii) $\mathcal{L}_{0}=\sum_{\alpha \in R^{\times}}\left[\mathcal{L}_{\alpha}, \mathcal{L}_{-\alpha}\right]$.

Example 2.5. Suppose that $I$ is a nonempty index set. Take $J:=\{0\} \uplus I \uplus \bar{I}$ and consider the $\mathbb{C}$-vector space $\mathcal{V}:=\mathcal{V}_{J}(\mathbb{C})$ as above. Define the bilinear form $(\cdot, \cdot)$ 
on $\mathcal{V}$ by

$$
\begin{aligned}
& \left(v_{j}, v_{\bar{k}}\right):=\left(v_{\bar{k}}, v_{j}\right):=2 \delta_{j, k}, \quad\left(v_{0}, v_{0}\right):=2, \\
& \left(v_{j}, v_{k}\right):=\left(v_{j}, v_{0}\right):=\left(v_{0}, v_{j}\right):=\left(v_{0}, v_{\bar{j}}\right):=\left(v_{\bar{j}}, v_{0}\right):=\left(v_{\bar{j}}, v_{\bar{k}}\right):=0
\end{aligned}
$$

for $j, k \in I$. For $\phi \in \operatorname{End}_{\mathbb{C}}(\mathcal{V})$, define $\bar{\phi} \in \operatorname{End}_{\mathbb{C}}(\mathcal{V})$ to be such that

$$
\text { if } \phi\left(v_{r}\right)=\sum_{j \in I} c_{j, r} v_{j} \text {, then } \bar{\phi}\left(v_{r}\right)=\sum_{j \in I} \bar{c}_{j, r} v_{j} \quad(r \in I)
$$

and set

$$
\mathfrak{L}:=\left\{\phi \in \mathfrak{g l}_{\mathbb{C}}(J) \mid(\phi(v), w)=-(v, \bar{\phi}(w)) \text { for all } v, w \in \mathcal{V}\right\} .
$$

Then $\mathfrak{L}$ is an $\mathbb{R}$-Lie subalgebra of $\mathfrak{g l}_{\mathbb{C}}(J)$. Take

$$
\mathcal{H}:=\operatorname{span}_{\mathbb{R}}\left\{h_{r}:=e_{r, r}-e_{\bar{r}, \bar{r}} \mid r \in I\right\}
$$

and for $r \in I$, define

$$
\epsilon_{r}: \mathcal{H} \rightarrow \mathbb{R}, \quad h_{j} \mapsto \delta_{r, j} \quad(j \in I) .
$$

Then $\mathfrak{L}$ has a weight space decomposition

$$
\mathfrak{L}=\bigoplus_{\alpha \in R} \mathfrak{L}_{\alpha}
$$

with respect to $\mathcal{H}$, where $R=\left\{0, \pm\left(\epsilon_{i} \pm \epsilon_{j}\right), \pm \epsilon_{i} \mid i \in I\right\}$ and

$$
\mathfrak{L}_{\alpha}= \begin{cases}\mathbb{R}\left(e_{0, \bar{r}}-e_{r, 0}\right)+i \mathbb{R}\left(e_{0, \bar{r}}+e_{r, 0}\right), & \alpha=\epsilon_{r}, \\ \mathbb{R}\left(e_{0, r}-e_{\bar{r}, 0}\right)+i \mathbb{R}\left(e_{0, r}+e_{\bar{r}, 0}\right), & \alpha=-\epsilon_{r}, \\ \mathbb{R}\left(e_{r, s}-e_{\bar{s}, \bar{r}}\right)+i \mathbb{R}\left(e_{r, s}+e_{\bar{s}, \bar{r}}\right), & \alpha=\epsilon_{r}-\epsilon_{s}, \\ \mathbb{R}\left(e_{r, \bar{s}}-e_{s, \bar{r}}\right)+i \mathbb{R}\left(e_{r, \bar{s}}+e_{s, \bar{r}}\right), & \alpha=\epsilon_{r}+\epsilon_{s}, \\ \mathbb{R}\left(e_{\bar{r}, s}-e_{\bar{s}, r}\right)+i \mathbb{R}\left(e_{\bar{r}, s}+e_{\bar{s}, r}\right), & \alpha=2 \epsilon_{r}, \\ \mathbb{R} i e_{r, \bar{r}}, & \alpha=-2 \epsilon_{r}, \\ \mathbb{R} i e_{\bar{r}, r}, & \alpha=0 . \\ i \mathbb{R} e_{0,0}+\sum_{r \in I} \mathbb{R}\left(e_{r, r}-e_{\bar{r}, \bar{r}}\right)+\sum_{r \in I} i \mathbb{R}\left(e_{r, r}+e_{\bar{r}, \bar{r}}\right), & \end{cases}
$$

Next set

$$
\mathcal{L}=\bigoplus_{\alpha \in R^{\times}} \mathfrak{L}_{\alpha} \oplus \sum_{\alpha \in R^{\times}}\left[\mathfrak{L}_{\alpha}, \mathfrak{L}_{-\alpha}\right] .
$$

Then $\mathcal{L}$ has a weight space decomposition $\mathcal{L}=\bigoplus_{\alpha \in R} \mathcal{L}_{\alpha}$ with respect to $\mathcal{H}$, in which

$$
\mathcal{L}_{\alpha}= \begin{cases}\mathfrak{L}_{\alpha}, & \alpha \in R^{\times}, \\ \sum_{\beta \in R^{\times}}\left[\mathfrak{L}_{\beta}, \mathfrak{L}_{-\beta}\right], & \alpha=0,\end{cases}
$$


Also it is easy to see that

$$
\mathcal{G}:=\bigoplus_{r, s \in I} \mathbb{R}\left(e_{r, s}-e_{\bar{s}, \bar{r}}\right) \oplus \bigoplus_{r, s \in I} i \mathbb{R}\left(e_{r, \bar{s}}+e_{s, \bar{r}}\right) \oplus \bigoplus_{r, s \in I} i \mathbb{R}\left(e_{\bar{r}, s}+e_{\bar{s}, r}\right)
$$

is an $\mathbb{R}$-subalgebra of $\mathcal{L}$. One can see that $\varphi: \mathcal{G} \rightarrow \mathfrak{s p}_{\mathbb{R}}(I)$ mapping

$$
\sum_{r, s \in I} a_{r, s}\left(e_{r, s}-e_{\bar{s}, \bar{r}}\right)+\sum_{r, s \in I} i b_{r, s}\left(e_{r, \bar{s}}+e_{s, \bar{r}}\right)+\sum_{r, s \in I} i c_{r, s}\left(e_{\bar{r}, s}+e_{\bar{s}, r}\right)
$$

to

$$
\sum_{r, s \in I} a_{r, s}\left(e_{r, s}-e_{\bar{s}, \bar{r}}\right)+\sum_{r, s \in I} b_{r, s}\left(e_{r, \bar{s}}+e_{s, \bar{r}}\right)-\sum_{r, s \in I} c_{r, s}\left(e_{\bar{r}, s}+e_{\bar{s}, r}\right),
$$

is a Lie algebra isomorphism. In particular, $\mathcal{G}$ is a locally finite split simple Lie subalgebra of $\mathcal{L}$ of type $C_{I}$ with splitting Cartan subalgebra $\mathcal{H}$. Altogether, $\mathcal{L}$ is a Lie algebra graded by $R$ which is a locally finite root system of type $B C_{I}$.

In [19], the author studies Lie algebras graded by an infinite irreducible locally finite root system which is not necessarily reduced and gives a complete description of such Lie algebras. The ingredients involved in this description for an $R$-graded Lie algebra is a locally finite split simple Lie algebra $\mathcal{G}$ of type $R_{\text {sdiv }}$, certain $\mathcal{G}$ modules and a quadruple called coordinate quadruple. In what follows we first recall what we mean by a coordinate quadruple and then state the recognition theorems for root graded Lie algebras as in [19].

By a star algebra $(\mathfrak{A}, \star)$, we mean an algebra $\mathfrak{A}$ together with an involution $\star$, that is, an antiautomorphism with $\star^{2}=\mathrm{id}_{\mathfrak{A}}$.

We call a quadruple $(\mathfrak{a}, *, \mathcal{C}, f)$ a coordinate quadruple if one of the following cases holds:

- (Type $A$ ) $\mathfrak{a}$ is a unital associative algebra, $*=\operatorname{id}_{\mathfrak{a}}, \mathcal{C}=\{0\}$ and $f: \mathcal{C} \times \mathcal{C} \rightarrow \mathfrak{a}$ is the zero map.

- (Type $B$ ) $\mathfrak{a}=\mathcal{A} \oplus \mathcal{B}$ where $\mathcal{A}$ is a unital commutative associative algebra, $\mathcal{B}$ is a unital associative $\mathcal{A}$-module equipped with a symmetric bilinear form and $\mathfrak{a}$ is the corresponding Clifford Jordan algebra [16], $*$ is a linear transformation fixing the elements of $\mathcal{A}$ and skew-fixing the elements of $\mathcal{B}, \mathcal{C}=\{0\}$ and $f: \mathcal{C} \times \mathcal{C} \rightarrow \mathfrak{a}$ is the zero map.

- (Type $C$ ) $\mathfrak{a}$ is a unital associative algebra, $*$ is an involution on $\mathfrak{a}, \mathcal{C}=\{0\}$ and $f: \mathcal{C} \times \mathcal{C} \rightarrow \mathfrak{a}$ is the zero map.

- (Type $D$ ) $\mathfrak{a}$ is a unital commutative associative algebra, $*=\mathrm{id}_{\mathfrak{a}}, \mathcal{C}=\{0\}$ and $f: \mathcal{C} \times \mathcal{C} \rightarrow \mathfrak{a}$ is the zero map.

- (Type $B C$ ) $\mathfrak{a}$ is a unital associative algebra, $*$ is an involution on $\mathfrak{a}, \mathcal{C}$ is a unital associative $\mathfrak{a}$-module and $f: \mathcal{C} \times \mathcal{C} \rightarrow \mathfrak{a}$ is a skew-hermitian form. 
Suppose that $\mathfrak{q}:=(\mathfrak{a}, *, \mathcal{C}, f)$ is a coordinate quadruple. Denote by $\mathcal{A}$ and $\mathcal{B}$ the fixed and the skew-fixed points of $\mathfrak{a}$ under $*$, respectively. Set $\mathfrak{b}:=\mathfrak{b}(\mathfrak{q}):=\mathfrak{a} \oplus \mathcal{C}$ and define

$$
\begin{aligned}
& \cdot: \mathfrak{b} \times \mathfrak{b} \rightarrow \mathfrak{b}, \\
& \left(\alpha_{1}+c_{1}, \alpha_{2}+c_{2}\right) \mapsto\left(\alpha_{1} \cdot \alpha_{2}\right)+f\left(c_{1}, c_{2}\right)+\alpha_{1} \cdot c_{2}+\alpha_{2}^{*} \cdot c_{1},
\end{aligned}
$$

for $\alpha_{1}, \alpha_{2} \in \mathfrak{a}$ and $c_{1}, c_{2} \in \mathcal{C}$. Then $(\mathfrak{b}, \cdot)$ is an algebra. For $\beta, \beta^{\prime} \in \mathfrak{b}$, set

$$
\beta \circ \beta^{\prime}:=\beta \cdot \beta^{\prime}+\beta^{\prime} \cdot \beta \text { and }\left[\beta, \beta^{\prime}\right]:=\beta \cdot \beta^{\prime}-\beta^{\prime} \cdot \beta,
$$

and for $c, c^{\prime} \in \mathcal{C}$, define

$$
\begin{aligned}
& \diamond: \mathcal{C} \times \mathcal{C} \rightarrow \mathcal{A}, \quad\left(c, c^{\prime}\right) \mapsto \frac{f\left(c, c^{\prime}\right)-f\left(c^{\prime}, c\right)}{2}, \\
& \diamond: \mathcal{C} \times \mathcal{C} \rightarrow \mathcal{B}, \quad\left(c, c^{\prime}\right) \mapsto \frac{f\left(c, c^{\prime}\right)+f\left(c^{\prime}, c\right)}{2} .
\end{aligned}
$$

For $\gamma=a+b+c \in \mathfrak{b}$ and $\gamma^{\prime}=a^{\prime}+b^{\prime}+c^{\prime} \in \mathfrak{b}$ with $a, a^{\prime} \in \mathcal{A}, b, b^{\prime} \in \mathcal{B}$ and $c, c^{\prime} \in \mathcal{C}$, set

$$
\beta_{\gamma, \gamma^{\prime}}^{*}:=\left[a, a^{\prime}\right]+\left[b, b^{\prime}\right]-c \circ c^{\prime}, \quad \gamma^{*}:=c .
$$

Now suppose that $\ell$ is a positive integer and for $\gamma, \gamma^{\prime} \in \mathfrak{b}$ consider the endomorphisms

$$
\begin{aligned}
& d_{\gamma, \gamma^{\prime}}^{\ell}: \mathfrak{b} \rightarrow \mathfrak{b}, \\
& \eta \mapsto \begin{cases}\left.\frac{1}{\ell+1}\left[\gamma, \gamma^{\prime}\right], \eta\right], & \mathfrak{q} \text { of type } A, \eta \in \mathfrak{b}, \\
\gamma^{\prime}(\gamma \eta)-\gamma\left(\gamma^{\prime} \eta\right), & \mathfrak{q} \text { of type } B, \eta \in \mathfrak{b}, \\
\frac{1}{2 \ell}\left[\beta_{\gamma, \gamma^{\prime}}^{*}, \eta\right] & \mathfrak{q} \text { of type } C \text { or } B C, \eta \in \mathfrak{a}, \\
\frac{1}{2 \ell}\left(\beta_{\gamma, \gamma^{\prime}}^{*} \cdot \eta\right)-\frac{1}{2}\left(f\left(\eta, \gamma^{\prime *}\right) \cdot \gamma^{*}+f\left(\eta, \gamma^{*}\right) \cdot \gamma^{\prime *}\right) \\
0, & \mathfrak{q} \text { of type } C \text { or } B C, \eta \in \mathcal{C},\end{cases}
\end{aligned}
$$

One can see that for $\gamma, \gamma^{\prime} \in \mathfrak{b}, d_{\gamma, \gamma^{\prime}}^{\ell}$ is a derivation of the algebra $\mathfrak{b}$. Next take $K$ to be the subspace of $\mathfrak{b} \otimes \mathfrak{b}$ spanned by

$$
\begin{aligned}
& \alpha \otimes c, \quad c \otimes \alpha, \quad a \otimes b, \\
& \alpha \otimes \alpha^{\prime}+\alpha^{\prime} \otimes \alpha, \quad c \otimes c^{\prime}-c^{\prime} \otimes c, \\
& \left(\alpha \cdot \alpha^{\prime}\right) \otimes \alpha^{\prime \prime}+\left(\alpha^{\prime \prime} \cdot \alpha\right) \otimes \alpha^{\prime}+\left(\alpha^{\prime} \cdot \alpha^{\prime \prime}\right) \otimes \alpha, \\
& f\left(c, c^{\prime}\right) \otimes \alpha+\left(\alpha^{*} \cdot c^{\prime}\right) \otimes c-(\alpha \cdot c) \otimes c^{\prime}
\end{aligned}
$$


for $\alpha, \alpha^{\prime}, \alpha^{\prime \prime} \in \mathfrak{a}, a \in \mathcal{A}, b \in \mathcal{B}$, and $c, c^{\prime} \in \mathcal{C}$. Then $\{\mathfrak{b}, \mathfrak{b}\}:=(\mathfrak{b} \otimes \mathfrak{b}) / K$ is a Lie algebra under the Lie bracket

$$
\begin{aligned}
& {\left[\left(\beta_{1} \otimes \beta_{2}\right)+K\right.}\left.,\left(\beta_{1}^{\prime} \otimes \beta_{2}^{\prime}\right)+K\right] \\
&\left.:=\left(\left(d_{\beta_{1}, \beta_{2}}^{\ell}\left(\beta_{1}^{\prime}\right) \otimes \beta_{2}^{\prime}\right)+K\right)+\left(\beta_{1}^{\prime} \otimes d_{\beta_{1}, \beta_{2}}^{\ell}\left(\beta_{2}^{\prime}\right)\right)+K\right)
\end{aligned}
$$

for $\beta_{1}, \beta_{2}, \beta_{1}^{\prime}, \beta_{2}^{\prime} \in \mathfrak{b}$ (see [3, Prop. 5.23] and [2]). We denote this Lie algebra by $\{\mathfrak{b}, \mathfrak{b}\}_{\ell}$ and for $\beta_{1}, \beta_{2} \in \mathfrak{b}$, we denote $\left(\beta_{1} \otimes \beta_{2}\right)+K$ by $\left\{\beta_{1}, \beta_{2}\right\}_{\ell}$ (or $\left\{\beta_{1}, \beta_{2}\right\}$ if there is no confusion). We recall the full skew-dihedral homology group

$$
\mathrm{HF}(\mathfrak{b}):=\left\{\sum_{i=1}^{n}\left\{\beta_{i}, \beta_{i}^{\prime}\right\} \in\{\mathfrak{b}, \mathfrak{b}\} \mid \sum_{i=1}^{n} d_{\beta_{i}, \beta_{i}^{\prime}}^{\ell}=0\right\}
$$

of $\mathfrak{b}$ (with respect to $\ell$ ) from [3] and [2], and note that it is a subset of the center of $\{\mathfrak{b}, \mathfrak{b}\}_{\ell}$. We say a subset $\mathcal{K}$ of the full skew-dihedral homology group of $\mathfrak{b}$ has the uniform property on $\mathfrak{b}$ if for $\beta_{1}, \beta_{1}^{\prime}, \ldots, \beta_{n}, \beta_{n}^{\prime} \in \mathfrak{b}$,

$$
\sum_{i=1}^{n}\left\{\beta_{i}, \beta_{i}^{\prime}\right\}_{\ell} \in \mathcal{K} \quad \text { implies that } \sum_{i=1}^{n} \beta_{\beta_{i}, \beta_{i}^{\prime}}^{*}=0 .
$$

We set

$$
\begin{aligned}
& A(\mathfrak{b}):=\left\{\sum_{i=1}^{n}\left\{\beta_{i}, \beta_{i}^{\prime}\right\} \in\{\mathfrak{b}, \mathfrak{b}\} \mid \sum_{i=1}^{n} \beta_{\beta_{i}, \beta_{i}^{\prime}}^{*}=0\right\} \\
& B(\mathfrak{b}):=\left\{\sum_{i=1}^{n}\left\{\beta_{i}, \beta_{i}^{\prime}\right\} \in\{\mathfrak{b}, \mathfrak{b}\} \mid \sum_{i=1}^{n}\left(f\left(\beta, \beta_{i}^{\prime *}\right) \cdot \beta_{i}^{*}+f\left(\beta, \beta_{i}^{*}\right) \cdot \beta_{i}^{\prime *}\right)=0 \forall \beta \in \mathfrak{b}\right\}
\end{aligned}
$$

and notice that

$$
\begin{aligned}
& \text { A subset } K \subseteq\{\mathfrak{b}, \mathfrak{b}\} \text { has the uniform property on } \mathfrak{b} \\
& \text { if and only if } K \subseteq \mathfrak{U}(\mathfrak{b}):=A(\mathfrak{b}) \cap B(\mathfrak{b}) \text {. }
\end{aligned}
$$

Now we recall Theorems 4.3, 4.4 and 4.1 from [19].

Theorem 2.6 (Recognition theorem for types $A$ and $D$ ). Suppose that $I$ is an infinite index set and $I_{0}$ is a subset of $I$ of cardinality $\ell>5$. Let $R$ be an irreducible locally finite root system of type $X=\dot{A}_{I}$ or $D_{I}$. Suppose that $\mathcal{V}$ is a vector space with a basis $\left\{v_{i} \mid i \in I\right\}$ and take $\mathcal{G}$ to be the locally finite split simple Lie algebra of type $X$. Define

$$
\mathfrak{I}_{0}: \mathcal{V} \rightarrow \mathcal{V}, \quad v_{i} \mapsto \begin{cases}v_{i}, & i \in I_{0}, \\ 0, & \text { otherwise }\end{cases}
$$


Also for $x, y \in \mathcal{G}$, define

$$
x \circ y:=x y+y x-\frac{2 \operatorname{tr}(x y)}{l+1} \mathfrak{I}_{0} .
$$

Suppose that $\left(\mathcal{A}, \operatorname{id}_{\mathcal{A}},\{0\}, \mathbf{0}\right)$ is a coordinate quadruple of type $X$ and $\mathcal{K} \subseteq \mathfrak{U}(\mathcal{A})$. Set

$$
\mathcal{L}(\mathcal{A}, \mathcal{K}):=(\mathcal{G} \otimes \mathcal{A}) \oplus\langle\mathcal{A}, \mathcal{A}\rangle
$$

where $\langle\mathcal{A}, \mathcal{A}\rangle$ is the quotient space $\{\mathcal{A}, \mathcal{A}\}_{\ell} / \mathcal{K}$ and for a, $a^{\prime} \in \mathcal{A}$, take $\left\langle a, a^{\prime}\right\rangle:=$ $\left\{a, a^{\prime}\right\}_{\ell}+\mathcal{K}$. Then considering $(2.16), \mathcal{L}(\mathcal{A}, \mathcal{K})$ together with

$$
\begin{aligned}
& {\left[x \otimes a, y \otimes a^{\prime}\right]= \begin{cases}{[x, y] \otimes \frac{1}{2}\left(a \circ a^{\prime}\right)+(x \circ y) \otimes \frac{1}{2}\left[a, a^{\prime}\right]+\operatorname{tr}(x y)\left\langle a, a^{\prime}\right\rangle,} & X=\dot{A}_{I}, \\
{[x, y] \otimes a a^{\prime}+\operatorname{tr}(x y)\left\langle a, a^{\prime}\right\rangle,} & X=D_{I},\end{cases} } \\
& {\left[\left\langle a_{1}, a_{2}\right\rangle, x \otimes a\right]= \begin{cases}-\frac{1}{2(\ell+1)}\left(\left(x \circ \mathfrak{I}_{0}\right) \otimes\left[a,\left[a_{1}, a_{2}\right]\right]\right. \\
\left.+\left[x, \mathfrak{I}_{0}\right] \otimes\left(a \circ\left[a_{1}, a_{2}\right]\right)+2 \operatorname{tr}\left(\mathfrak{I}_{0} x\right)\left\langle a,\left[a_{1}, a_{2}\right]\right\rangle\right), & X=\dot{A}_{I}, \\
0, & X=D_{I},\end{cases} } \\
& {\left[\left\langle a_{1}, a_{2}\right\rangle,\left\langle a_{1}^{\prime}, a_{2}^{\prime}\right\rangle\right]= \begin{cases}\left\langle d_{a_{1}, a_{2}}^{\ell}\left(a_{1}^{\prime}\right), a_{2}^{\prime}\right\rangle+\left\langle a_{1}^{\prime}, d_{a_{1}, a_{2}}^{\ell}\left(a_{2}^{\prime}\right)\right\rangle, & X=\dot{A}_{I}, \\
0, & X=D_{I},\end{cases} }
\end{aligned}
$$

for $x, y \in \mathcal{G}, a, a^{\prime}, a_{1}, a_{2}, a_{1}^{\prime}, a_{2}^{\prime} \in \mathcal{A}$, is a Lie algebra graded by $R$ with grading pair $(\mathcal{G}, \mathcal{H})$ where $\mathcal{H}$ is defined as in (2.3) or (2.11). Moreover, up to isomorphism any $R$-graded Lie algebra arises in this manner.

Theorem 2.7 (Recognition theorem for types $B$ and $C$ ). Suppose that $I$ is an infinite index set and $I_{0}$ is a subset of $I$ of cardinality $\ell>4$. Take $\mathcal{G}$ to be either $\mathfrak{o}_{B}(I)$ or $\mathfrak{s p}(I)$. Suppose that $\mathcal{V}$ is a vector space with a basis $\left\{v_{0}, v_{i}, v_{\bar{i}} \mid\right.$ $i \in I\}$ equipped with a nondegenerate symmetric bilinear form $(\cdot, \cdot)$ as in (2.4) if $\mathcal{G}=\mathfrak{o}_{B}(I)$ and it is a vector space with a basis $\left\{v_{i}, v_{\bar{i}} \mid i \in I\right\}$ equipped with a nondegenerate skew-symmetric bilinear form $(\cdot, \cdot)$ as in $(2.6)$ if $\mathcal{G}=\mathfrak{s p}(I)$. Set

$$
T:= \begin{cases}I_{0} \cup \bar{I}_{0} \cup\{0\} & \text { if } \mathcal{G}=\mathfrak{o}_{B}(I), \\ I_{0} \cup \overline{I_{0}} & \text { if } \mathcal{G}=\mathfrak{s p}(I),\end{cases}
$$

and define $\mathfrak{I}_{0}: \mathcal{V} \rightarrow \mathcal{V}$ to be the linear transformation defined by

$$
v_{i} \mapsto \begin{cases}v_{i} & \text { if } i \in T, \\ 0 & \text { if } i \in I \cup \bar{I} \backslash T .\end{cases}
$$

Next set $\mathcal{S}:=\mathcal{V}$ if $\mathcal{G}=\mathfrak{o}_{B}(I)$ and take $\mathcal{S}$ as in $(2.9)$ if $\mathcal{G}=\mathfrak{s p}(I)$. For e, $f \in \mathcal{G} \cup \mathcal{S}$, set

$$
e \circ f:=e f+f e-\frac{\operatorname{tr}(e f)}{\ell} \Im_{0},
$$


and for $u, v \in \mathcal{V}$, define $D_{u, v} \in \operatorname{End}(\mathcal{V})$ as follows:

$$
D_{u, v}: \mathcal{V} \rightarrow \mathcal{V}, \quad w \mapsto(u, w) v-(v, w) u, \quad w \in \mathcal{V}
$$

Suppose that $R$ is an irreducible locally finite root system of type $X=B_{I}$ or $C_{I}$ and $(\mathfrak{a}, *, \mathcal{C}, f)$ is a coordinate quadruple of type $X$. Take $\mathcal{A}$ and $\mathcal{B}$ to be the set of $*$-fixed and $*$-skew-fixed points of $\mathfrak{a}$ respectively. For a subset $\mathcal{K}$ of $\mathfrak{U}(\mathfrak{a})$, set

$$
\mathcal{L}(\mathfrak{a}, \mathcal{K}):=(\mathcal{G} \otimes \mathcal{A}) \oplus(\mathcal{S} \otimes \mathcal{B}) \oplus\langle\mathfrak{a}, \mathfrak{a}\rangle,
$$

where $\langle\mathfrak{a}, \mathfrak{a}\rangle$ is the quotient space $\{\mathfrak{a}, \mathfrak{a}\}_{\ell} / \mathcal{K}$, and for $\alpha, \alpha^{\prime} \in \mathfrak{a}$, take $\left\langle\alpha, \alpha^{\prime}\right\rangle:=$ $\left\{\alpha, \alpha^{\prime}\right\}_{\ell}+\mathcal{K}$. Then considering $(2.16), \mathcal{L}(\mathfrak{a}, \mathcal{K})$ together with

$$
\begin{aligned}
& {\left[x \otimes a, y \otimes a^{\prime}\right]=[x, y] \otimes a a^{\prime}+\operatorname{tr}(x y)\left\langle a, a^{\prime}\right\rangle,} \\
& {[x \otimes a, s \otimes b]=x s \otimes a b} \\
& {\left[s \otimes b, t \otimes b^{\prime}\right]=D_{s, t} \otimes f\left(b, b^{\prime}\right)+(s, t)\left\langle b, b^{\prime}\right\rangle} \\
& {\left[\left\langle\alpha_{1}, \alpha_{2}\right\rangle, x \otimes a\right]=x \otimes d_{\alpha_{1}, \alpha_{2}}^{\ell}(a),} \\
& {\left[\left\langle\alpha_{1}, \alpha_{2}\right\rangle, s \otimes b\right]=s \otimes d_{\alpha_{1}, \alpha_{2}}^{\ell}(b),} \\
& {\left[\left\langle\alpha_{1}, \alpha_{2}\right\rangle,\left\langle\alpha_{1}^{\prime}, \alpha_{2}^{\prime}\right\rangle\right]=\left\langle d_{\alpha_{1}, \alpha_{2}}^{\ell}\left(\alpha_{1}^{\prime}\right), \alpha_{2}^{\prime}\right\rangle+\left\langle\alpha_{1}, d_{\alpha_{1}^{\prime}, \alpha_{2}^{\prime}}^{\ell}\left(\alpha_{2}\right)\right\rangle}
\end{aligned}
$$

for $x, y \in \mathcal{G}, s, t \in \mathcal{S}, \alpha, \alpha^{\prime}, \alpha_{1}, \alpha_{2}, \alpha_{1}^{\prime}, \alpha_{2}^{\prime} \in \mathfrak{a}, a, a^{\prime} \in \mathcal{A}$ and $b, b^{\prime} \in \mathcal{B}$ if $\mathcal{G}=\mathfrak{o}_{B}(I)$, and

$$
\begin{aligned}
& {\left[x \otimes a, y \otimes a^{\prime}\right]=[x, y] \otimes \frac{1}{2}\left(a \circ a^{\prime}\right)+(x \circ y) \otimes \frac{1}{2}\left[a, a^{\prime}\right]+\operatorname{tr}(x y)\left\langle a, a^{\prime}\right\rangle,} \\
& {[x \otimes a, s \otimes b]=(x \circ s) \otimes \frac{1}{2}[a, b]+[x, s] \otimes \frac{1}{2}(a \circ b),} \\
& {\left[s \otimes b, t \otimes b^{\prime}\right]=[s, t] \otimes \frac{1}{2}\left(b \circ b^{\prime}\right)+(s \circ t) \otimes \frac{1}{2}\left[b, b^{\prime}\right]+\operatorname{tr}(s t)\left\langle b, b^{\prime}\right\rangle,} \\
& {\left[\left\langle\alpha, \alpha^{\prime}\right\rangle, x \otimes a\right]=\frac{-1}{4 \ell}\left(\left(x \circ \mathfrak{I}_{0}\right) \otimes\left[a, \beta_{\alpha, \alpha^{\prime}}^{*}\right]+\left[x, \mathfrak{I}_{0}\right] \otimes\left(a \circ \beta_{\alpha, \alpha^{\prime}}^{*}\right)\right),} \\
& {\left[\left\langle\alpha, \alpha^{\prime}\right\rangle, s \otimes b\right]=\frac{-1}{4 \ell}\left(\left[s, \mathfrak{I}_{0}\right] \otimes\left(b \circ \beta_{\alpha_{1}, \alpha_{2}}^{*}\right)+\left(s \circ \mathfrak{I}_{0}\right) \otimes\left[b, \beta_{\alpha_{1}, \alpha_{2}}^{*}\right]+2 \operatorname{tr}\left(s \mathfrak{I}_{0}\right)\left\langle b, \beta_{\alpha, \alpha^{\prime}}^{*}\right\rangle\right),} \\
& {\left[\left\langle\alpha_{1}, \alpha_{2}\right\rangle,\left\langle\alpha_{1}^{\prime}, \alpha_{2}^{\prime}\right\rangle\right]=\left\langle d_{\alpha_{1}, \alpha_{2}}^{\ell}\left(\alpha_{1}^{\prime}\right), \alpha_{2}^{\prime}\right\rangle+\left\langle\alpha_{1}^{\prime}, d_{\alpha_{1}, \alpha_{2}}^{\ell}\left(\alpha_{2}^{\prime}\right)\right\rangle}
\end{aligned}
$$

(see (2.15)) for $x, y \in \mathcal{G}, s, t \in \mathcal{S}, a, a^{\prime} \in \mathcal{A}, b, b^{\prime} \in \mathcal{B}, \alpha, \alpha^{\prime}, \alpha_{1}, \alpha_{2}, \alpha_{1}^{\prime}, \alpha_{2}^{\prime} \in \mathfrak{a}$ if $\mathcal{G}=\mathfrak{s p}(I)$, is a Lie algebra graded by $R$ with grading pair $(\mathcal{G}, \mathcal{H})$ where $\mathcal{H}$ is defined as in (2.5) or (2.7). Moreover, up to isomorphism any $R$-graded Lie algebra arises in this manner.

Theorem 2.8 (Recognition theorem for type $B C$ ). Suppose that $I$ is an infinite index set and $I_{0}$ is a fixed subset of $I$ of cardinality $\ell>3$. Assume that $R$ is an irreducible locally finite root system of type $B C_{I}$ and $\mathcal{V}$ is a vector space with a basis $\left\{v_{i} \mid i \in I \cup \bar{I}\right\}$. Suppose that $(\cdot, \cdot)$ is a bilinear form as in (2.6), set $\mathcal{G}:=\mathfrak{s} \mathfrak{p}(I)$ 
and consider $\mathcal{S}$ as in (2.9). Define

$$
\mathfrak{I}_{0}: \mathcal{V} \rightarrow \mathcal{V}, \quad v_{i} \mapsto \begin{cases}v_{i}, & i \in I_{0} \cup \bar{I}_{0}, \\ 0, & \text { otherwise, }\end{cases}
$$

and for $e, f \in \mathcal{G} \cup \mathcal{S}$, define

$$
e \circ f:=e f+f e-\frac{\operatorname{tr}(e f)}{l} \mathfrak{I}_{0} .
$$

(i) Suppose that $(\mathfrak{a}, *, \mathcal{C}, f)$ is a coordinate quadruple of type $B C$ and $\mathcal{A}, \mathcal{B}$ are $*$-fixed and $*$-skew-fixed points of $\mathfrak{a}$ respectively. Set $\mathfrak{b}:=\mathfrak{b}(\mathfrak{a}, *, \mathcal{C}, f)$ and take $\circ,[\cdot, \cdot], \diamond, \diamond$ as in (2.13) and (2.14). For $\beta_{1}, \beta_{2} \in \mathfrak{b}$, consider $d_{\beta_{1}, \beta_{2}}^{\ell}$ as in (2.16) and take $\beta_{\beta_{1}, \beta_{2}}^{*}, \beta_{1}^{*}$ and $\beta_{2}^{*}$ as in $(2.15)$. For a subset $\mathcal{K}$ of $\mathfrak{U}(\mathfrak{b})$, set

$$
\mathcal{L}(\mathfrak{b}, \mathcal{K}):=(\mathcal{G} \otimes \mathcal{A}) \oplus(\mathcal{S} \otimes \mathcal{B}) \oplus(\mathcal{V} \otimes \mathcal{C}) \oplus\left(\{\mathfrak{b}, \mathfrak{b}\}_{\ell} / \mathcal{K}\right) .
$$

Then setting $\left\langle\beta, \beta^{\prime}\right\rangle:=\left\{\beta, \beta^{\prime}\right\}_{\ell}+\mathcal{K}, \beta, \beta^{\prime} \in \mathfrak{b}, \mathcal{L}(\mathfrak{b}, \mathcal{K})$ together with

$$
\begin{aligned}
& {\left[x \otimes a, y \otimes a^{\prime}\right]=[x, y] \otimes \frac{1}{2}\left(a \circ a^{\prime}\right)+(x \circ y) \otimes \frac{1}{2}\left[a, a^{\prime}\right]+\operatorname{tr}(x y)\left\langle a, a^{\prime}\right\rangle,} \\
& {[x \otimes a, s \otimes b]=(x \circ s) \otimes \frac{1}{2}[a, b]+[x, s] \otimes \frac{1}{2}(a \circ b)=-[s \otimes b, x \otimes a],} \\
& {\left[s \otimes b, t \otimes b^{\prime}\right]=[s, t] \otimes \frac{1}{2}\left(b \circ b^{\prime}\right)+(s \circ t) \otimes \frac{1}{2}\left[b, b^{\prime}\right]+\operatorname{tr}(s t)\left\langle b, b^{\prime}\right\rangle,} \\
& {[x \otimes a, u \otimes c]=x u \otimes a \cdot c=-[u \otimes c, x \otimes a],} \\
& {[s \otimes b, u \otimes c]=s u \otimes b \cdot c=-[u \otimes c, s \otimes b],} \\
& {\left[u \otimes c, v \otimes c^{\prime}\right]=(u \circ v) \otimes\left(c \otimes c^{\prime}\right)+[u, v] \otimes\left(c \odot c^{\prime}\right)+(u, v)\left\langle c, c^{\prime}\right\rangle,} \\
& {\left[\left\langle\beta_{1}, \beta_{2}\right\rangle, x \otimes a\right]=\frac{-1}{4 \ell}\left(\left(x \circ \mathfrak{I}_{0}\right) \otimes\left[a, \beta_{\beta_{1}, \beta_{2}}^{*}\right]+\left[x, \Im_{0}\right] \otimes\left(a \circ \beta_{\beta_{1}, \beta_{2}}^{*}\right)\right),} \\
& {\left[\left\langle\beta_{1}, \beta_{2}\right\rangle, s \otimes b\right]=\frac{-1}{4 \ell}\left(\left[s, \Im_{0}\right] \otimes\left(b \circ \beta_{\beta_{1}, \beta_{2}}^{*}\right)+\left(s \circ \Im_{0}\right) \otimes\left[b, \beta_{\beta_{1}, \beta_{2}}^{*}\right]+2 \operatorname{tr}\left(s \Im_{0}\right)\left\langle b, \beta_{\beta_{1}, \beta_{2}}^{*}\right\rangle\right),} \\
& {\left[\left\langle\beta_{1}, \beta_{2}\right\rangle, v \otimes c\right]=\frac{1}{2 \ell} \Im_{0} v \otimes\left(\beta_{\beta_{1}, \beta_{2}}^{*} c\right)-\frac{1}{2} v \otimes\left(f\left(c, \beta_{2}^{*}\right) \cdot \beta_{1}^{*}+f\left(c, \beta_{1}^{*}\right) \cdot \beta_{2}^{*}\right),} \\
& {\left[\left\langle\beta_{1}, \beta_{2}\right\rangle,\left\langle\beta_{1}^{\prime}, \beta_{2}^{\prime}\right\rangle\right]=\left\langle d_{\beta_{1}, \beta_{2}}\left(\beta_{1}^{\prime}\right), \beta_{2}^{\prime}\right\rangle+\left\langle\beta_{1}^{\prime}, d_{\beta_{1}, \beta_{2}}^{\ell}\left(\beta_{2}^{\prime}\right)\right\rangle}
\end{aligned}
$$

for $x, y \in \mathcal{G}, s, t \in \mathcal{S}, u, v \in \mathcal{V}, a, a^{\prime} \in \mathcal{A}, b, b^{\prime} \in \mathcal{B}, c, c^{\prime} \in \mathcal{C}, \beta_{1}, \beta_{2}, \beta_{1}^{\prime}, \beta_{2}^{\prime} \in \mathfrak{b}$ is an $R$-graded Lie algebra with grading pair $(\mathcal{G}, \mathcal{H})$ where $\mathcal{H}$ is the splitting Cartan subalgebra of $\mathcal{G}$ defined in (2.7).

(ii) If $\mathcal{L}$ is an $R$-graded Lie algebra with grading pair $(\mathfrak{g}, \mathfrak{h})$, then there is a coordinate quadruple $(\mathfrak{a}, *, \mathcal{C}, f)$ of type $B C$ and a subspace $\mathcal{K}$ of $\mathfrak{U}(\mathfrak{b})$ for $\mathfrak{b}:=$ $\mathfrak{b}(\mathfrak{a}, *, \mathcal{C}, f)$ such that $\mathcal{L}$ is isomorphic to $\mathcal{L}(\mathfrak{b}, \mathcal{K})$.

Remark 2.9. (i) $[19$, Rem. 4.2] The Lie algebra $\mathcal{L}(\mathfrak{b}, \mathcal{K})$ does not depend on the choice of $\ell$ and $I_{0}$.

(ii) Suppose that $\ell$ is an integer greater than 5 and consider coordinate quadruples $\mathfrak{q}_{1}=\left(\mathfrak{a}_{1}, *_{1}, \mathcal{C}_{1}, f_{1}\right)$ and $\mathfrak{q}_{2}=\left(\mathfrak{a}_{2}, *_{2}, \mathcal{C}_{2}, f_{2}\right)$. We say $\mathfrak{q}_{1}$ and $\mathfrak{q}_{2}$ are isomorphic if they are of the same type and there is an algebra isomorphism $\varphi: \mathfrak{a}_{1} \rightarrow \mathfrak{a}_{2}$ and also a linear isomorphism $\psi: \mathcal{C}_{1} \rightarrow \mathcal{C}_{2}$ such that

$$
*_{2} \circ \varphi=\varphi \circ *_{1}, \quad f_{2}\left(\psi(c), \psi\left(c^{\prime}\right)\right)=\varphi\left(f_{1}\left(c, c^{\prime}\right)\right) \quad \text { and } \quad \psi(\alpha \cdot c)=\varphi(\alpha) \cdot \psi(c)
$$


for $\alpha \in \mathfrak{a}$ and $c, c^{\prime} \in \mathcal{C}$. We note that $\varphi \oplus \psi$ induces a Lie algebra isomorphism $\theta_{\varphi, \psi}$ from $\left\{\mathfrak{b}\left(\mathfrak{q}_{1}\right), \mathfrak{b}\left(\mathfrak{q}_{1}\right)\right\}_{\ell}$ to $\left\{\mathfrak{b}\left(\mathfrak{q}_{2}\right), \mathfrak{b}\left(\mathfrak{q}_{2}\right)\right\}_{\ell}$.

For subspaces $\mathcal{K}_{1}$ of $\operatorname{HF}\left(\mathfrak{b}\left(\mathfrak{q}_{1}\right)\right)$ and $\mathcal{K}_{2}$ of $\operatorname{HF}\left(\mathfrak{b}\left(\mathfrak{q}_{2}\right)\right)$ satisfying the uniform property on $\mathfrak{b}\left(\mathfrak{q}_{1}\right)$ and $\mathfrak{b}\left(\mathfrak{q}_{2}\right)$ respectively, we say $\left(\mathfrak{q}_{1}, \mathcal{K}_{1}\right)$ is isomorphic to $\left(\mathfrak{q}_{2}, \mathcal{K}_{2}\right)$ if $\mathfrak{q}_{1}$ is isomorphic to $\mathfrak{q}_{2}$ via a pair $(\varphi, \psi)$ as above and $\theta_{\varphi, \psi}\left(\mathcal{K}_{1}\right)=\mathcal{K}_{2}$. It is not difficult to prove that $\left(\mathfrak{q}_{1}, \mathcal{K}_{1}\right)$ is isomorphic to $\left(\mathfrak{q}_{2}, \mathcal{K}_{2}\right)$ if and only if the Lie algebras $\mathcal{L}\left(\mathfrak{q}_{1}, \mathcal{K}_{1}\right)$ and $\mathcal{L}\left(\mathfrak{q}_{2}, \mathcal{K}_{2}\right)$ are isomorphic. This means that for a root graded Lie algebra $\mathcal{L}$, up to isomorphism, there is a unique coordinate quadruple; we refer to as the corresponding coordinate quadruple of $\mathcal{L}$.

\section{$\S 3$. Central extensions}

In this section, we study central extensions of a Lie algebra graded by an infinite irreducible locally finite root system and give a complete description of them. In particular, we describe the universal central extension of a root graded Lie algebra. Throughout this section, we denote by $Z(\mathcal{L})$ the center of a Lie algebra $\mathcal{L}$. Also for a map $f: A \rightarrow B$ and $C \subseteq A$, by $\left.f\right|_{C}$ we mean the restriction of $f$ to $C$.

A Lie algebra epimorphism $\pi: \tilde{\mathcal{L}} \rightarrow \mathcal{L}$ from $(\tilde{\mathcal{L}},[\cdot, \cdot] \sim)$ to $(\mathcal{L},[\cdot, \cdot])$ is called a central extension of $\mathcal{L}$ if $C:=\operatorname{ker}(\pi) \subseteq Z(\tilde{\mathcal{L}})$. We may always assume that $\tilde{\mathcal{L}}=\mathcal{L} \oplus C$ and that $[x+e, y+f]^{\sim}=[x, y]+\tau(x, y)$ for $x, y \in \mathcal{L}$ and $e, f \in C$, where $\tau: \mathcal{L} \times \mathcal{L} \rightarrow C$ is a 2-cocycle. In this case, $\pi: \tilde{\mathcal{L}} \rightarrow \mathcal{L}$ is the canonical projection map. The central extension $\pi$ is called perfect if $\tilde{\mathcal{L}}$ is a perfect Lie algebra.

Lemma 3.1. Suppose that $\mathcal{L}$ is a Lie algebra and $\mathfrak{g}$ is a finite-dimensional simple Lie subalgebra of $\mathcal{L}$. Let $\tau: \mathcal{L} \times \mathcal{L} \rightarrow C$ be a 2 -cocycle and $\left(\tilde{\mathcal{L}}:=\mathcal{L} \oplus C,[\cdot, \cdot]^{\sim}\right)$ the corresponding central extension. Consider $\tilde{\mathcal{L}}$ as a $\mathfrak{g}$-module via the action

$$
\cdot: \mathfrak{g} \times \tilde{\mathcal{L}} \rightarrow \tilde{\mathcal{L}}, \quad(x, y) \mapsto[x, y]^{\sim}, \quad x \in \mathfrak{g}, y \in \tilde{\mathcal{L}} .
$$

If $D$ is a trivial $\mathfrak{g}$-submodule of $\mathcal{L}$ via the adjoint representation, then $D$ is a trivial $\mathfrak{g}$-submodule of $\tilde{\mathcal{L}}$, in particular $\tau(\mathfrak{g}, D)=\{0\}$.

Proof. For $d_{1}, \ldots, d_{n} \in D$ and $r_{1}, \ldots, r_{n} \in \tau(\mathfrak{g}, D)$,

$$
\operatorname{span}_{\mathbb{F}}\left\{d_{1}, \ldots, d_{n}\right\}+\sum_{i=1}^{n} \tau\left(\mathfrak{g}, d_{i}\right)+\operatorname{span}_{\mathbb{F}}\left\{r_{1}, \ldots, r_{n}\right\}
$$

is a finite-dimensional $\mathcal{G}$-submodule of $\tilde{\mathcal{L}}$. This implies that $D \oplus \tau(\mathfrak{g}, D)$ is a locally finite $\mathfrak{g}$-submodule of $\tilde{\mathcal{L}}$. A generalization of Weyl's Theorem implies that $D \oplus$ $\tau(\mathfrak{g}, D)$ is a completely reducible $\mathfrak{g}$-module. Now as $\tau(\mathfrak{g}, D)$ is a $\mathfrak{g}$-submodule of 
$\mathcal{D} \oplus \tau(\mathfrak{g}, \mathcal{D})$, there is a submodule $\dot{D}$ of $D \oplus \tau(\mathfrak{g}, D)$ such that $D \oplus \tau(\mathfrak{g}, D)=$ $\dot{D} \oplus \tau(\mathfrak{g}, D)$. Since $\dot{D}$ is a $\mathfrak{g}$-submodule of $D \oplus \tau(\mathfrak{g}, D)$, we have

$$
[\mathfrak{g}, D]^{\sim} \subseteq[\mathfrak{g}, \dot{D} \oplus \tau(\mathfrak{g}, D)]^{\sim} \subseteq[\mathfrak{g}, \dot{D}]^{\sim} \subseteq \dot{D} .
$$

But $D$ is a trivial $\mathfrak{g}$-submodule of $\mathcal{L}$ and so $[\mathfrak{g}, D]^{\sim} \subseteq \tau(\mathfrak{g}, D)$. Therefore $[\mathfrak{g}, D]^{\sim} \subseteq$ $\dot{D} \cap \tau(\mathfrak{g}, D)=\{0\}$. This completes the proof.

Lemma 3.2. Suppose that $R$ is an irreducible locally finite root system and $\mathcal{L}=$ $\bigoplus_{\alpha \in R} \mathcal{L}_{\alpha}$ is an $R$-graded Lie algebra with grading pair $(\mathfrak{g}, \mathcal{H})$. Suppose $\tau: \mathcal{L} \times \mathcal{L}$ $\rightarrow C$ is a 2-cocycle satisfying $\tau(\mathcal{L}, \mathfrak{g})=\{0\}$. Consider the corresponding central extension $\pi:\left(\tilde{\mathcal{L}},[\cdot, \cdot]^{\sim}\right) \rightarrow \mathcal{L}$ and suppose $\tilde{\mathcal{L}}$ is perfect. Then $\tilde{\mathcal{L}}$ is an R-graded Lie algebra with grading pair $(\mathfrak{g}, \mathcal{H})$ and weight space decomposition $\tilde{\mathcal{L}}=\bigoplus_{\alpha \in R} \tilde{\mathcal{L}}_{\alpha}$ in which

$$
\tilde{\mathcal{L}}_{\alpha}= \begin{cases}\mathcal{L}_{\alpha} & \text { if } \alpha \in R \backslash\{0\} \\ \mathcal{L}_{0} \oplus C & \text { if } \alpha=0\end{cases}
$$

Moreover, if $R$ is an irreducible finite root system, then the coordinate quadruple of $\mathcal{L}$ coincides with the coordinate quadruple of $\tilde{\mathcal{L}}$.

Proof. The first assertion follows from an easy verification. So we suppose $R$ is an irreducible finite root system and prove the second assertion. We know that the Lie algebra epimorphism $\pi: \tilde{\mathcal{L}} \rightarrow \mathcal{L}$ induces a Lie algebra epimorphism $\varphi$ : $\tilde{\mathcal{L}} / Z(\tilde{\mathcal{L}}) \rightarrow \mathcal{L} / Z(\mathcal{L})$ mapping $\tilde{x}+Z(\tilde{\mathcal{L}})$ to $\pi(\tilde{x})+Z(\mathcal{L})$ for $\tilde{x} \in \tilde{\mathcal{L}}$. We claim that $\varphi$ is a Lie algebra isomorphism. Suppose that $\tilde{x} \in \tilde{\mathcal{L}}$ and $\tilde{x}+Z(\tilde{\mathcal{L}}) \in \operatorname{ker}(\varphi)$. Therefore $\pi(\tilde{x}) \in Z(\mathcal{L})$ and so for each $\tilde{y} \in \tilde{\mathcal{L}}, \pi\left([\tilde{x}, \tilde{y}]^{\sim}\right)=[\pi(\tilde{x}), \pi(\tilde{y})]=0$. This implies that $[\tilde{x}, \tilde{y}]^{\sim} \in \operatorname{ker}(\pi) \subseteq Z(\tilde{\mathcal{L}})$ for all $\tilde{y} \in \tilde{\mathcal{L}}$. But $\tilde{\mathcal{L}}$ is perfect, so one concludes that $\tilde{x} \in Z(\tilde{\mathcal{L}})$. Thus $\varphi$ is injective. Next we note that $\mathcal{L}$ and $\tilde{\mathcal{L}}$ are perfect and $\varphi$ is an isomorphism, so $\mathcal{L}, \mathcal{L} / Z(\mathcal{L}), \tilde{\mathcal{L}}$ and $\tilde{\mathcal{L}} / Z(\tilde{\mathcal{L}})$ have the same universal central extension, say $\mathfrak{A}$. Therefore $\mathcal{L}$ as well as $\tilde{\mathcal{L}}$ are quotient algebras of $\mathfrak{A}$ by subspaces of the center of $\mathfrak{A}$. Now we conclude using [2, Thm. 4.20] and [3, Thm. 5.34].

We want to study central extensions of root graded Lie algebras. Let us start with type $B C$. From now on, we assume $R$ is an irreducible locally finite root system of type $B C_{I}$ for an infinite index set $I$. With the notation as in Section 1, we set

$$
J:=I \cup \bar{I}, \quad \mathcal{V}:=\mathcal{V}_{J}, \quad \mathcal{G}:=\mathfrak{s p}(I) .
$$

We know that $\mathcal{G}=\sum_{\alpha \in R_{\text {sdiv }}} \mathcal{G}_{\alpha}$ is a locally finite split simple Lie algebra of type $C_{I}$ with splitting Cartan subalgebra $\mathcal{H}$ as in (2.7). Fix a finite subset $I_{0}$ of $I$ of cardinality $\ell$ greater than 3 and suppose $\left\{I_{\lambda} \mid \lambda \in \Lambda\right\}$, where $\Lambda$ is an index set containing a symbol named zero, is the class of all finite subsets of $I$ containing $I_{0}$. 
For $\lambda, \mu \in \Lambda$, we say $\lambda \preccurlyeq \mu$ if $I_{\lambda} \subset I_{\mu}$. For $\lambda \in \Lambda$, suppose $R_{\lambda}$ is a finite subsystem of $R$ of type $B C_{I_{\lambda}}$ and take

$$
\mathcal{G}^{\lambda}:=\sum_{\alpha \in\left(R_{\lambda}\right)_{\text {sdiv }}^{\times}} \mathcal{G}_{\alpha} \oplus \sum_{\alpha \in\left(R_{\lambda}\right)_{\text {sdiv }}^{\times}}\left[\mathcal{G}_{\alpha}, \mathcal{G}_{-\alpha}\right] .
$$

Then $\mathcal{G}^{\lambda}$ is a finite-dimensional split simple Lie subalgebra of $\mathcal{G}$ of type $\left(R_{\lambda}\right)_{\text {sdiv }}$ and $\mathcal{H}_{\lambda}:=\mathcal{G}^{\lambda} \cap \mathcal{H}$ is a splitting Cartan subalgebra of $\mathcal{G}^{\lambda}$. Next we recall the irreducible $\mathcal{G}$-module $\mathcal{S}$ from $(2.9)$ and set

$$
\mathcal{V}^{\lambda}:=\operatorname{span}_{\mathbb{F}}\left\{v_{r} \mid r \in I_{\lambda} \cup \bar{I}_{\lambda}\right\}, \quad \mathcal{S}^{\lambda}:=\mathcal{S} \cap \operatorname{span}_{\mathbb{F}}\left\{e_{r, s} \mid r, s \in I_{\lambda} \cup \bar{I}_{\lambda}\right\}
$$

Then $\mathcal{V}^{\lambda}$ and $\mathcal{S}^{\lambda}$ are irreducible finite-dimensional $\mathcal{G}^{\lambda}$-modules with the set of weights $\left(R_{\lambda}\right)_{\mathrm{sh}}$ and $\{0\} \cup\left(R_{\lambda}\right)_{\lg }$ respectively. We note that $\mathcal{G}$ is the direct union of $\left\{\mathcal{G}^{\lambda} \mid \lambda \in \Lambda\right\}, \mathcal{V}$ is the direct union of $\left\{\mathcal{V}^{\lambda} \mid \lambda \in \Lambda\right\}$ and $\mathcal{S}$ is the direct union of $\left\{\mathcal{S}^{\lambda} \mid \lambda \in \Lambda\right\}$ (here, the direct union is, by definition, the direct limit of a direct system whose morphisms are inclusion maps).

Now suppose that $\mathfrak{q}=(\mathfrak{a}, *, \mathcal{C}, f)$ is a coordinate quadruple of type $B C$, take $\mathfrak{b}:=\mathfrak{b}(\mathfrak{q})$ to be the algebra corresponding to $\mathfrak{q}$ as in Section 1, and suppose $\mathcal{K} \subseteq \mathfrak{U}(\mathfrak{b})$. Take $\mathcal{A}$ and $\mathcal{B}$ to be the $*$-fixed and $*$-skew-fixed points of $\mathfrak{a}$ respectively and consider the $R$-graded Lie algebra

$$
\mathcal{L}:=\mathcal{L}(\mathfrak{q}, \mathcal{K})=(\mathcal{G} \otimes \mathcal{A}) \oplus(\mathcal{S} \otimes \mathcal{B}) \oplus(\mathcal{V} \otimes \mathcal{C}) \oplus\langle\mathfrak{b}, \mathfrak{b}\rangle
$$

with grading pair $(\mathcal{G}, \mathcal{H})$ as in Theorem 2.8. We next recall from $[19, \S 4]$ that for $\lambda \in \Lambda$, there is a subalgebra $\mathcal{D}_{\lambda}$ of $\mathcal{L}$ with $\mathcal{D}_{0}=\langle\mathfrak{b}, \mathfrak{b}\rangle$ such that

$$
\begin{aligned}
& {\left[\mathcal{G}^{\lambda}, \mathcal{D}_{\lambda}\right]=\{0\}} \\
& \mathcal{D}_{\lambda} \oplus\left(\mathcal{S}^{\lambda} \otimes \mathcal{B}\right)=\mathcal{D}_{0} \oplus\left(\mathcal{S}^{\lambda} \otimes \mathcal{B}\right) \\
& \mathcal{L}^{\lambda}:=\left(\mathcal{G}^{\lambda} \otimes \mathcal{A}\right) \oplus\left(\mathcal{S}^{\lambda} \otimes \mathcal{B}\right) \oplus\left(\mathcal{V}^{\lambda} \otimes \mathcal{C}\right) \oplus \mathcal{D}_{\lambda} \\
& \quad=\left(\mathcal{G}^{\lambda} \otimes \mathcal{A}\right) \oplus\left(\mathcal{S}^{\lambda} \otimes \mathcal{B}\right) \oplus\left(\mathcal{V}^{\lambda} \otimes \mathcal{C}\right) \oplus \mathcal{D}_{0}
\end{aligned}
$$

where $\mathcal{L}^{\lambda}$ is a Lie algebra graded by $R_{\lambda}$.

Now suppose that $\pi:\left(\tilde{\mathcal{L}},[\cdot, \cdot]^{\sim}\right) \rightarrow(\mathcal{L},[\cdot, \cdot])$ is a central extension of $\mathcal{L}$ with corresponding 2-cocycle $\tau$. We note that

$$
\begin{aligned}
\tilde{\mathcal{L}}^{\lambda} & :=\left(\mathcal{G}^{\lambda} \otimes \mathcal{A}\right) \oplus\left(\mathcal{S}^{\lambda} \otimes \mathcal{B}\right) \oplus\left(\mathcal{V}^{\lambda} \otimes \mathcal{C}\right) \oplus \mathcal{D}_{\lambda} \oplus \operatorname{ker}(\pi) \\
& =\left(\mathcal{G}^{\lambda} \otimes \mathcal{A}\right) \oplus\left(\mathcal{S}^{\lambda} \otimes \mathcal{B}\right) \oplus\left(\mathcal{V}^{\lambda} \otimes \mathcal{C}\right) \oplus \mathcal{D}_{0} \oplus \operatorname{ker}(\pi)
\end{aligned}
$$

is a central extension of $\mathcal{L}^{\lambda}$. Regard $\tilde{\mathcal{L}}$ as a $\mathcal{G}$-module via the action

$$
\cdot: \mathcal{G} \times \tilde{\mathcal{L}} \rightarrow \tilde{\mathcal{L}}, \quad(x, y) \mapsto[x, y]^{\sim}, \quad x \in \mathcal{G}, y \in \tilde{\mathcal{L}}
$$


and note that $\pi$ is a $\mathcal{G}$-module homomorphism. Next for $\lambda \in \Lambda$, we define the subalgebra

$$
\mathcal{E}_{\lambda}:=\mathcal{G}^{\lambda} \oplus \tau(\mathcal{G}, \mathcal{G})
$$

of $\tilde{\mathcal{L}}$. One can see that $\mathcal{E}_{\lambda}$ is a $\mathcal{G}^{\lambda}$-module via the action "." restricted to $\mathcal{G}^{\lambda} \times \mathcal{E}_{\lambda}$. Now for a positive integer $n, x_{1}, \ldots, x_{n} \in \mathcal{G}^{\lambda}$ and $r_{1}, \ldots, r_{n} \in \tau(\mathcal{G}, \mathcal{G})$, the set $\left\{x_{1}+r_{1}, \ldots, x_{n}+r_{n} \mid 1 \leq i \leq n\right\}$ is a subset of

$$
\mathcal{G}^{\lambda} \oplus\left(\tau\left(\mathcal{G}^{\lambda}, \mathcal{G}^{\lambda}\right)+\operatorname{span}_{\mathbb{F}}\left\{r_{1}, \ldots, r_{n}\right\}\right),
$$

which is a finite-dimensional $\mathcal{G}^{\lambda}$-submodule of $\mathcal{E}_{\lambda}$. This implies that $\mathcal{E}_{\lambda}$ is a locally finite $\mathcal{G}^{\lambda}$-module. Therefore it is completely reducible as $\mathcal{G}^{\lambda}$ is a finite-dimensional split simple Lie algebra. Now as $\tau(\mathcal{G}, \mathcal{G})$ is a $\mathcal{G}^{0}$-submodule of $\mathcal{E}_{0}$, there is a $\mathcal{G}^{0}$ submodule $\dot{\mathcal{G}}^{0}$ of $\mathcal{E}_{0}$ such that $\mathcal{E}_{0}=\dot{\mathcal{G}}^{0} \oplus \tau(\mathcal{G}, \mathcal{G})$. We next observe that $\mathcal{E}_{0} \subseteq \mathcal{E}_{\lambda}$ for $\lambda \in \Lambda$, and define

$$
\dot{\mathcal{G}}^{\lambda}:=\text { the } \mathcal{G}^{\lambda} \text {-submodule of } \mathcal{E}_{\lambda}(\subseteq \tilde{\mathcal{L}}) \text { generated by } \dot{\mathcal{G}}^{0} .
$$

Lemma 3.3. (i) Set $\mathcal{E}:=\mathcal{G} \oplus \tau(\mathcal{G}, \mathcal{G})$. Then $\mathcal{E}$ is a Lie subalgebra of $\tilde{\mathcal{L}}$ and also $a \mathcal{G}$-submodule of $\tilde{\mathcal{L}}$. Moreover, the restriction of $\pi$ to $\mathcal{E}$ is both a Lie algebra homomorphism and $a \mathcal{G}$-module homomorphism.

(ii) Consider (3.7). For $\lambda \in \Lambda$, we have the following:

(a) $\dot{\mathcal{G}}^{\lambda}$ is a Lie subalgebra of $\tilde{\mathcal{L}}$.

(b) The restriction of $\pi$ to $\dot{\mathcal{G}}^{\lambda}$ is a $\mathcal{G}^{\lambda}$-module isomorphism from $\dot{\mathcal{G}}^{\lambda}$ onto $\mathcal{G}^{\lambda}$. In particular, $\dot{\mathcal{G}}^{\lambda}$ is an irreducible $\mathcal{G}^{\lambda}$-submodule of $\mathcal{E}_{\lambda}\left(\subseteq \tilde{\mathcal{L}}_{\lambda}\right)$ isomorphic to $\mathcal{G}^{\lambda}$.

(c) $\mathcal{G}^{\lambda} \oplus \tau(\mathcal{G}, \mathcal{G})=\dot{\mathcal{G}}^{\lambda} \oplus \tau(\mathcal{G}, \mathcal{G})$.

(d) The restriction of $\pi$ to $\dot{\mathcal{G}}^{\lambda}$ is a Lie algebra isomorphism from $\dot{\mathcal{G}}^{\lambda}$ onto $\mathcal{G}^{\lambda}$. In particular, $\dot{\mathcal{G}}^{\lambda}$ is a Lie subalgebra of $\mathcal{E}_{\lambda}\left(\subseteq \tilde{\mathcal{L}}_{\lambda}\right)$ isomorphic to $\mathcal{G}^{\lambda}$.

Proof. (i) This is easy to see.

(ii) If $\lambda=0$, we get the result using the fact that $\mathcal{G}^{0} \oplus \tau(\mathcal{G}, \mathcal{G})=\dot{\mathcal{G}}^{0} \oplus \tau(\mathcal{G}, \mathcal{G})$. Now we assume $\lambda \in \Lambda$ with $\lambda \neq 0$ and prove (a)-(d) simultaneously. We know that $\mathcal{E}_{\lambda}=\mathcal{G}^{\lambda} \oplus \tau(\mathcal{G}, \mathcal{G})$ is a locally finite $\mathcal{G}^{\lambda}$-submodule of $\tilde{\mathcal{L}}$ under the action "." restricted to $\mathcal{G}^{\lambda} \times \mathcal{E}_{\lambda}$ and that $\tau(\mathcal{G}, \mathcal{G})$ is a submodule of $\mathcal{G}^{\lambda} \oplus \tau(\mathcal{G}, \mathcal{G})$. Therefore, there is a $\mathcal{G}^{\lambda}$-submodule $\mathcal{P}$ of $\mathcal{G}^{\lambda} \oplus \tau(\mathcal{G}, \mathcal{G})$ such that $\mathcal{E}_{\lambda}=\mathcal{G}^{\lambda} \oplus \tau(\mathcal{G}, \mathcal{G})=\mathcal{P} \oplus$ $\tau(\mathcal{G}, \mathcal{G})$. It follows that $\mathcal{P}$ is a subalgebra of $\tilde{\mathcal{L}}$ and that $\theta:=\left.\pi\right|_{\mathcal{P}}: \mathcal{P} \rightarrow \mathcal{G}^{\lambda}$ is a Lie algebra isomorphism as well as a $\mathcal{G}^{\lambda}$-module isomorphism. Consider the weight space decomposition

$$
\mathcal{G}^{\lambda}=\sum_{\alpha \in\left(R_{\lambda}\right)_{\text {sdiv }}}\left(\mathcal{G}^{\lambda}\right)_{\alpha}
$$


for $\mathcal{G}^{\lambda}$ with respect to $\mathcal{H}^{\lambda}$ and the weight space decomposition

$$
\mathcal{G}^{0}=\sum_{\alpha \in\left(R_{0}\right)_{\text {sdiv }}}\left(\mathcal{G}^{0}\right)_{\alpha}
$$

for $\mathcal{G}^{0}$ with respect to $\mathcal{H}_{0}$. Then

$$
\left(\mathcal{G}^{0}\right)_{\alpha}= \begin{cases}\left(\mathcal{G}^{\lambda}\right)_{\alpha}, & \alpha \in\left(R_{0}\right)_{\text {sdiv }}^{\times} \\ \sum_{\alpha \in\left(R_{0}\right)_{\text {sdiv }}^{\times}}\left[\left(\mathcal{G}^{\lambda}\right)_{\alpha},\left(\mathcal{G}^{\lambda}\right)_{-\alpha}\right], & \alpha=0 .\end{cases}
$$

We next set

$$
\begin{cases}\mathcal{P}_{\alpha}:=\theta^{-1}\left(\left(\mathcal{G}^{\lambda}\right)_{\alpha}\right), & \alpha \in\left(R_{\lambda}\right)_{\text {sdiv }} \\ \mathcal{Q}_{\alpha}:=\theta^{-1}\left(\left(\mathcal{G}^{0}\right)_{\alpha}\right), & \alpha \in\left(R_{0}\right)_{\text {sdiv }}\end{cases}
$$

Then $\mathcal{P}=\mathcal{P}_{0} \oplus \sum_{\alpha \in\left(R_{\lambda}\right)_{\text {sdiv }}^{\times}} \mathcal{P}_{\alpha}$ is a weight space decomposition for the $\mathcal{G}^{\lambda}$ module $\mathcal{P}$ with respect to $\mathcal{H}_{\lambda}$. Moreover $\mathcal{Q}:=\theta^{-1}\left(\mathcal{G}^{0}\right)$ is a $\mathcal{G}^{0}$-submodule of $\mathcal{P}$ isomorphic to $\mathcal{G}^{0}$ and it is equipped with the weight space decomposition $\mathcal{Q}=$ $\sum_{\alpha \in\left(R_{0}\right)_{\text {sdiv }}} \mathcal{Q}_{\alpha}$ with respect to $\mathcal{H}_{0}$. Using this together with part (ii)(b) for $\lambda=0$, we have:

- $\left(\mathcal{P}_{0} \oplus \tau(\mathcal{G}, \mathcal{G})\right) \oplus \sum_{\alpha \in\left(R_{\lambda}\right)_{\text {sdiv }}^{\times}} \mathcal{P}_{\alpha}$ is a weight space decomposition for $\mathcal{E}_{\lambda}$ with respect to $\mathcal{H}_{\lambda}$,

- $\left(\mathcal{Q}_{0} \oplus \tau(\mathcal{G}, \mathcal{G})\right) \oplus \sum_{\alpha \in\left(R_{0}\right)_{\text {sdiv }}^{\times}} \mathcal{Q}_{\alpha}$ is a weight space decomposition for $\mathcal{E}_{0}=\mathcal{Q} \oplus$ $\tau(\mathcal{G}, \mathcal{G})$ with respect to $\mathcal{H}_{0}$

- $\dot{\mathcal{G}}^{0}$ is a nontrivial finite-dimensional irreducible $\mathcal{G}^{0}$-submodule of $\mathcal{E}_{0}$ isomorphic to $\mathcal{G}^{0}$.

So by [19, Prop. 2.15], $\dot{\mathcal{G}}^{\lambda}$ is a $\mathcal{G}^{\lambda}$-submodule of $\mathcal{E}_{\lambda}$ isomorphic to $\mathcal{G}^{\lambda}$. It is not difficult to see that the restriction of $\pi$ to $\dot{\mathcal{G}}^{\lambda}$ is a $\mathcal{G}^{\lambda}$-module isomorphism from $\dot{\mathcal{G}}^{\lambda}$ onto $\mathcal{G}^{\lambda}$. This in turn implies that $\dot{\mathcal{G}}^{\lambda} \oplus \tau(\mathcal{G}, \mathcal{G})=\mathcal{G}^{\lambda} \oplus \tau(\mathcal{G}, \mathcal{G})$. Now as $\dot{\mathcal{G}}^{\lambda}$ is a $\mathcal{G}^{\lambda}$-submodule of $\tilde{\mathcal{L}}$, one finds that $\dot{\mathcal{G}}^{\lambda}$ is a subalgebra of $\tilde{\mathcal{L}}$ and that $\left.\pi\right|_{\dot{\mathcal{G}}_{\lambda}}: \dot{\mathcal{G}}^{\lambda} \rightarrow \mathcal{G}^{\lambda}$ is a Lie algebra isomorphism.

Corollary 3.4. (i) For $\lambda, \mu \in \Lambda$ with $\lambda \preccurlyeq \mu$, we have $\dot{\mathcal{G}}^{\lambda} \subseteq \dot{\mathcal{G}}^{\mu}$, in particular $\bigcup_{\lambda \in \Lambda} \dot{\mathcal{G}}^{\lambda}$ is a subalgebra of $\tilde{\mathcal{L}}$ and also a $\mathcal{G}$-submodule of $\overline{\tilde{\mathcal{L}}}$.

(ii) Set $\dot{\mathcal{G}}:=\bigcup_{\lambda \in \Lambda} \dot{\mathcal{G}}^{\lambda}$. Then $\left.\pi\right|_{\dot{\mathcal{G}}}$ is a Lie algebra isomorphism as well as a $\mathcal{G}$-module isomorphism from $\dot{\mathcal{G}}$ onto $\mathcal{G}$.

(iii) $\mathcal{G} \oplus \tau(\mathcal{G}, \mathcal{G})=\dot{\mathcal{G}} \oplus \tau(\mathcal{G}, \mathcal{G})$.

Proof. Since $\dot{\mathcal{G}}^{0} \subseteq \dot{\mathcal{G}}^{\lambda} \cap \dot{\mathcal{G}}^{\mu}$ and $\mathcal{G}^{\lambda} \subseteq \mathcal{G}^{\mu}$, we have $\dot{\mathcal{G}}^{\lambda} \subseteq \dot{\mathcal{G}}^{\mu}$. Now using Proposition 3.3(ii), we are done. 
Recall (3.3) and suppose $\mathcal{I}$ is an index set containing a symbol named zero. Fix a basis $\left\{a_{i} \mid i \in \mathcal{I}\right\}$ with $a_{0}=1$ for $\mathcal{A}$, a basis $\left\{b_{j} \mid j \in \mathcal{J}\right\}$ for $\mathcal{B}$ and a basis $\left\{c_{t} \mid t \in \mathcal{T}\right\}$ for $\mathcal{C}$. For $i \in \mathcal{I}, j \in \mathcal{J}$ and $t \in \mathcal{T}$, set

$$
\mathcal{G}_{i}:=\mathcal{G} \otimes a_{i}, \quad \mathcal{S}_{j}:=\mathcal{S} \otimes b_{j}, \quad \mathcal{V}_{t}:=\mathcal{V} \otimes c_{t} ;
$$

note that these are $\mathcal{G}$-submodules of $\mathcal{L}$. We identify $\mathcal{G} \otimes 1$ with $\mathcal{G}$. Now for $\lambda \in \Lambda$, set

$$
\mathcal{G}_{i}^{\lambda}:=\mathcal{G}^{\lambda} \otimes a_{i}, \quad \mathcal{S}_{j}^{\lambda}:=\mathcal{S}^{\lambda} \otimes b_{j}, \quad \mathcal{V}_{t}^{\lambda}:=\mathcal{V}^{\lambda} \otimes c_{t}
$$

Suppose $\mathcal{M}$ is one of the $\mathcal{G}$-submodules of $\mathcal{L}$ in the class $\left\{\mathcal{G}_{i}, \mathcal{S}_{j}, \mathcal{V}_{t} \mid i \in \mathcal{I} \backslash\{0\}\right.$, $j \in \mathcal{J}, t \in \mathcal{T}\}$, and for $\lambda \in \Lambda$, consider $\mathcal{M}^{\lambda}$ as in (3.9). By [19, Prop. 2.12(b)] the vector space $\mathcal{M}$ is the direct union of the class $\left\{\mathcal{M}^{\lambda} \mid \lambda \in \Lambda\right\}$ where for each $\lambda \in \Lambda$,

- $\mathcal{M}^{\lambda}$ is a finite-dimensional irreducible $\mathcal{G}^{\lambda}$-submodule of $\mathcal{L}^{\lambda}$,

- $\mathcal{M}^{\lambda}$ has a weight space decomposition with respect to $\mathcal{H}_{\lambda}$, whose set of weights, $\Gamma_{\lambda}$, can be identified as a subset of $R_{\lambda}$,

- for $\mu \in \Lambda$ with $\lambda \preccurlyeq \mu$, we have $\Gamma_{\lambda} \subseteq \Gamma_{\mu}$ and $\left(\mathcal{M}^{\lambda}\right)_{\gamma}=\left(\mathcal{M}^{\mu}\right)_{\gamma}$ for all $\gamma \in \Gamma_{\lambda} \backslash\{0\}$,

- $\Gamma_{\lambda} \backslash\{0\}= \begin{cases}\left(R_{\lambda}\right)_{\mathrm{sh}} & \text { if } \Gamma_{0} \backslash\{0\}=\left(R_{0}\right)_{\mathrm{sh}}, \\ \left(R_{\lambda}\right)_{\mathrm{sdiv}}^{\times} & \text {if } \Gamma_{0} \backslash\{0\}=\left(R_{0}\right)_{\mathrm{sdiv}}^{\times}, \\ \left(\left(R_{\lambda}\right)_{\mathrm{sdiv}}\right)_{\mathrm{sh}} & \text { if } \Gamma_{0} \backslash\{0\}=\left(\left(R_{0}\right)_{\mathrm{sdiv}}\right)_{\mathrm{sh}} .\end{cases}$

Now set

$$
\tilde{\mathcal{M}}:=\mathcal{M} \oplus \tau(\mathcal{G}, \mathcal{M}) \quad \text { and } \quad \tilde{\mathcal{M}}^{\lambda}:=\mathcal{M}^{\lambda} \oplus \tau(\mathcal{G}, \mathcal{M}), \quad \lambda \in \Lambda
$$

We can see that for $\lambda \in \Lambda, \mathcal{M}^{\lambda}$ is a locally finite $\mathcal{G}^{\lambda}$-submodule of $\tilde{\mathcal{L}}$ under the action ". " restricted to $\mathcal{G}^{\lambda} \times \tilde{\mathcal{L}}$, and so it is completely reducible. This allows us to fix a $\mathcal{G}^{0}$-submodule $\dot{\mathcal{M}}^{0}$ of $\tilde{\mathcal{M}}^{0}$ such that

$$
\tilde{\mathcal{M}}^{0}=\dot{\mathcal{M}}^{0} \oplus \tau(\mathcal{G}, \mathcal{M})
$$

We next define

$$
\dot{\mathcal{M}}^{\lambda}:=\text { the } \mathcal{G}^{\lambda} \text {-submodule of } \tilde{\mathcal{M}}^{\lambda}(\subseteq \tilde{\mathcal{L}}) \text { generated by } \dot{\mathcal{M}}^{0}, \quad \lambda \in \Lambda .
$$

Lemma 3.5. (i) (a) For $\lambda \in \Lambda$, the restriction of $\pi$ to $\dot{\mathcal{M}}^{\lambda}$ is a $\mathcal{G}^{\lambda}$-module isomorphism from $\dot{\mathcal{M}}^{\lambda}$ onto $\mathcal{M}^{\lambda}$ and $\tilde{\mathcal{M}}^{\lambda}=\dot{\mathcal{M}}^{\lambda} \oplus \tau(\mathcal{G}, \mathcal{M})$.

(b) For $\lambda, \mu \in \Lambda$ with $\lambda \preccurlyeq \mu$, we have $\dot{\mathcal{M}}^{\lambda} \subseteq \dot{\mathcal{M}}^{\mu}$; in particular

$$
\dot{\mathcal{M}}:=\bigcup_{\lambda \in \Lambda} \dot{\mathcal{M}}^{\lambda}
$$

is a $\mathcal{G}$-submodule of $\tilde{\mathcal{L}}$. 
(c) The restriction of $\pi$ to $\dot{\mathcal{M}}$ is a $\mathcal{G}$-module isomorphism from $\dot{\mathcal{M}}$ onto $\mathcal{M}$ and $\tilde{\mathcal{M}}=\dot{\mathcal{M}} \oplus \tau(\mathcal{G}, \mathcal{M})=\mathcal{M} \oplus \tau(\mathcal{G}, \mathcal{M})$.

(ii) If $x \in \dot{\mathcal{M}}$ and $\pi(x) \in \mathcal{M}^{\lambda}$ for some $\lambda \in \Lambda$, then $x \in \dot{\mathcal{M}}^{\lambda}$.

Proof. (i) Consider (3.10) and use the same argument as in the proof of Proposition $3.3($ ii).

(ii) Take $\mu \in \Lambda$ such that $x \in \dot{\mathcal{M}}^{\mu}$. We know that there is $\nu \in \Lambda$ with $\lambda \preccurlyeq \nu$ and $\mu \preccurlyeq \nu$. Since the restriction of $\pi$ to $\dot{\mathcal{M}}^{\lambda}$ is a $\mathcal{G}^{\lambda}$-module isomorphism from $\dot{\mathcal{M}}^{\lambda}$ onto $\mathcal{M}^{\lambda}$, one can find $y \in \dot{\mathcal{M}}^{\lambda}$ such that $\pi(x)=\pi(y)$. So $x, y \in \dot{\mathcal{M}}^{\nu}$ and $\pi(x)=\pi(y)$. But the restriction of $\pi$ to $\dot{\mathcal{M}}^{\nu}$ is a $\mathcal{G}^{\nu}$-module isomorphism from $\dot{\mathcal{M}}^{\nu}$ onto $\mathcal{M}^{\nu}$, therefore $x=y \in \dot{\mathcal{M}}^{\lambda}$.

Consider (3.8) and (3.9) and recall that we identify $\mathcal{G} \otimes 1$ with $\mathcal{G}$. Suppose that $i \in \mathcal{I}, j \in \mathcal{J}$ and $t \in \mathcal{T}$. Then for $\lambda \in \Lambda$, contemplating (3.11) and using Propositions 3.5 and 3.3(ii), one gets:

- $\dot{\mathcal{G}}_{i}^{\lambda}$, the $\mathcal{G}^{\lambda}$-submodule of $\tilde{\mathcal{L}}$ generated by $\dot{\mathcal{G}}_{i}^{0}$, is an irreducible $\mathcal{G}^{\lambda}$-submodule isomorphic to $\mathcal{G}_{i}^{\lambda}$,

- $\dot{\mathcal{S}}_{j}^{\lambda}$, the $\mathcal{G}^{\lambda}$-submodule of $\tilde{\mathcal{L}}$ generated by $\dot{\mathcal{S}}_{j}^{0}$, is an irreducible $\mathcal{G}^{\lambda}$-submodule isomorphic to $\mathcal{S}_{j}^{\lambda}$,

- $\dot{\mathcal{V}}_{t}^{\lambda}$, the $\mathcal{G}^{\lambda}$-submodule of $\tilde{\mathcal{L}}$ generated by $\dot{\mathcal{V}}_{t}^{0}$, is an irreducible $\mathcal{G}^{\lambda}$-submodule isomorphic to $\mathcal{V}_{t}^{\lambda}$;

moreover setting

$$
\dot{\mathcal{G}}_{i}:=\underset{\lambda \in \Lambda}{\lim } \dot{\mathcal{G}}_{i}^{\lambda}, \quad \dot{\mathcal{S}}_{j}:=\underset{\lambda \in \Lambda}{\lim } \dot{\mathcal{S}}_{j}^{\lambda}, \quad \dot{\mathcal{V}}_{t}:=\underset{\lambda \in \Lambda}{\lim } \dot{\mathcal{V}}_{t}^{\lambda},
$$

we see that $\dot{\mathcal{G}}_{i}$ is a $\mathcal{G}$-submodule of $\tilde{\mathcal{L}}$ isomorphic to $\mathcal{G}_{i}, \dot{\mathcal{S}}_{j}$ is a $\mathcal{G}$-submodule of $\tilde{\mathcal{L}}$ isomorphic to $\mathcal{S}_{j}$, and $\dot{\mathcal{V}}_{t}$ is a $\mathcal{G}$-submodule of $\tilde{\mathcal{L}}$ isomorphic to $\mathcal{V}_{t}$. Finally, we have

$$
\begin{array}{rlrl}
\mathcal{G}_{i} \oplus \tau\left(\mathcal{G}, \mathcal{G}_{i}\right) & =\dot{\mathcal{G}}_{i} \oplus \tau\left(\mathcal{G}, \mathcal{G}_{i}\right), & & \mathcal{G}_{i}^{\lambda} \oplus \tau\left(\mathcal{G}, \mathcal{G}_{i}\right)=\dot{\mathcal{G}}_{i}^{\lambda} \oplus \tau\left(\mathcal{G}, \mathcal{G}_{i}\right), \\
\mathcal{S}_{j} \oplus \tau\left(\mathcal{G}, \mathcal{S}_{j}\right)=\dot{\mathcal{S}}_{j} \oplus \tau\left(\mathcal{G}, \mathcal{S}_{j}\right), & & \mathcal{S}_{j}^{\lambda} \oplus \tau\left(\mathcal{G}, \mathcal{S}_{j}\right)=\dot{\mathcal{S}}_{j}^{\lambda} \oplus \tau\left(\mathcal{G}, \mathcal{S}_{j}\right), \\
\mathcal{V}_{t} \oplus \tau\left(\mathcal{G}, \mathcal{V}_{t}\right)=\dot{\mathcal{V}}_{t} \oplus \tau\left(\mathcal{G}, \mathcal{V}_{t}\right), & & \mathcal{V}_{t}^{\lambda} \oplus \tau\left(\mathcal{G}, \mathcal{V}_{t}\right)=\dot{\mathcal{V}}_{t}^{\lambda} \oplus \tau\left(\mathcal{G}, \mathcal{V}_{t}\right)
\end{array}
$$

Lemma 3.6. (i) Recall (3.4); there is a subspace $\dot{\mathcal{D}}$ of $\left[\mathcal{L}^{0}, \mathcal{L}^{0}\right] \sim \cap\left(\mathcal{D}_{0} \oplus \operatorname{ker}(\pi)\right)$ such that

(1) $\pi(\dot{\mathcal{D}}) \subseteq \mathcal{D}_{0}$

(2) $\left.\pi\right|_{\dot{\mathcal{D}}}: \dot{\mathcal{D}} \rightarrow \mathcal{D}_{0}$ is a linear isomorphism, 
(3) $\left[\mathcal{G}^{0}, \dot{\mathcal{D}}\right]^{\sim}=\{0\}$,

(4) for $\lambda \in \Lambda,\left[\mathcal{G}^{\lambda}, \dot{\mathcal{D}}\right]^{\sim} \subseteq \sum_{j \in \mathcal{J}} \dot{\mathcal{S}}_{j}^{\lambda}$.

(ii) $\sum_{i \in \mathcal{I}} \dot{\mathcal{G}}_{i}+\sum_{j \in \mathcal{J}} \dot{\mathcal{S}}_{j}+\sum_{t \in \mathcal{T}} \dot{\mathcal{V}}_{t}+\dot{\mathcal{D}}$ is a direct sum.

Proof. (i) We note that $\mathcal{D}_{0} \subseteq \mathcal{L}^{0}=\left[\mathcal{L}^{0}, \mathcal{L}^{0}\right]$, so for $d \in \mathcal{D}_{0}$, there are $n \in \mathbb{N} \backslash\{0\}$ and $x_{i}, y_{i} \in \mathcal{L}^{0}, 1 \leq i \leq n$, such that $d=\sum_{i=1}^{n}\left[x_{i}, y_{i}\right]$. Therefore we have

$$
\sum_{i=1}^{n}\left[x_{i}, y_{i}\right]^{\sim}=d+\sum_{i=1}^{n} \tau\left(x_{i}, y_{i}\right) \in\left[\mathcal{L}^{0}, \mathcal{L}^{0}\right]^{\sim} \cap\left(\mathcal{D}_{0}+\operatorname{ker}(\pi)\right)
$$

and $\pi\left(\sum_{i=1}^{n}\left[x_{i}, y_{i}\right]^{\sim}\right)=d$. So one can find a subspace $\dot{\mathcal{D}}$ of $\left[\mathcal{L}^{0}, \mathcal{L}^{0}\right]^{\sim} \cap\left(\mathcal{D}_{0} \oplus \operatorname{ker}(\pi)\right)$ such that $\left.\pi\right|_{\mathcal{D}}: \dot{\mathcal{D}} \rightarrow \mathcal{D}_{0}$ is a linear isomorphism. Now using Lemma 3.1 together with the fact that $\left.\pi\right|_{\mathcal{L}^{0} \oplus \operatorname{ker}(\pi)}: \mathcal{L}^{0} \oplus \operatorname{ker}(\pi) \rightarrow \mathcal{L}^{0}$ is a central extension of $\mathcal{L}^{0}$, we deduce $\left[\mathcal{G}^{0}, \dot{\mathcal{D}}\right]^{\sim} \subseteq\left[\mathcal{G}^{0}, \mathcal{D}_{0}\right]^{\sim}=\{0\}$. So we get (1)-(3).

For (4), suppose $\lambda \in \Lambda, x \in \mathcal{G}^{\lambda}$ and $\dot{d} \in \dot{\mathcal{D}}$. Since $\mathcal{L}^{\lambda} \oplus \operatorname{ker}(\pi)$ is a central extension of $\mathcal{L}^{\lambda}$, using Lemma 3.1 together with (3.4) and (3.13), we have

$[x, \dot{d}]^{\sim} \in\left[x, \mathcal{D}_{0}+\operatorname{ker}(\pi)\right]^{\sim} \subseteq\left[x, \mathcal{D}_{\lambda}+\sum_{j \in \mathcal{J}} \mathcal{S}_{j}^{\lambda}+\operatorname{ker}(\pi)\right]^{\sim} \subseteq\left[x, \sum_{j \in \mathcal{J}} \dot{\mathcal{S}}_{j}^{\lambda}\right]^{\sim} \subseteq \sum_{j \in \mathcal{J}} \dot{\mathcal{S}}_{j}^{\lambda}$.

This completes the proof.

(ii) This is easy to see.

Thanks to Lemma 3.6(ii), we set

$$
\begin{aligned}
\dot{\mathcal{L}} & :=\bigoplus_{i \in \mathcal{I}} \dot{\mathcal{G}}_{i} \oplus \bigoplus_{j \in \mathcal{J}} \dot{\mathcal{S}}_{j} \oplus \bigoplus_{t \in \mathcal{T}} \dot{\mathcal{V}}_{t} \oplus \dot{\mathcal{D}}, \\
\dot{\mathcal{L}}^{\lambda} & :=\bigoplus_{i \in \mathcal{I}} \dot{\mathcal{G}}_{i}^{\lambda} \oplus \bigoplus_{j \in \mathcal{J}} \dot{\mathcal{S}}_{j}^{\lambda} \oplus \bigoplus_{t \in \mathcal{T}} \dot{\mathcal{V}}_{t}^{\lambda} \oplus \dot{\mathcal{D}}, \quad \lambda \in \Lambda .
\end{aligned}
$$

One can see that $\dot{\mathcal{L}} \cap \operatorname{ker}(\pi)=\{0\}$ and so by (3.13) and Lemma 3.6(i), we get

$$
\tilde{\mathcal{L}}=\dot{\mathcal{L}} \oplus \operatorname{ker}(\pi) .
$$

Also considering (3.5), (3.9) and (3.13) for $\lambda \in \Lambda$, we infer that

$$
\tilde{\mathcal{L}}^{\lambda}:=\mathcal{L}^{\lambda} \oplus \operatorname{ker}(\pi)=\dot{\mathcal{L}}^{\lambda} \oplus \operatorname{ker}(\pi)
$$

is a Lie subalgebra of $\tilde{\mathcal{L}}$, and $\tilde{\mathcal{L}}$ is the direct union of $\left\{\tilde{\mathcal{L}}^{\lambda} \mid \lambda \in \Lambda\right\}$.

Now take

$$
\pi_{1}: \tilde{\mathcal{L}} \rightarrow \dot{\mathcal{L}} \quad \text { and } \quad \pi_{2}: \tilde{\mathcal{L}} \rightarrow \operatorname{ker}(\pi)
$$


to be the projective maps on $\dot{\mathcal{L}}$ and $\operatorname{ker}(\pi)$ respectively, with respect to the decomposition $\tilde{\mathcal{L}}=\dot{\mathcal{L}} \oplus \operatorname{ker}(\pi)$, and for $x, y \in \dot{\mathcal{L}}$, define

$$
[x, y]^{\bullet}:=\pi_{1}\left([x, y]^{\sim}\right) \quad \text { and } \quad \dot{\tau}(x, y)=\pi_{2}\left([x, y]^{\sim}\right) .
$$

Then $\left(\dot{\mathcal{L}},[\cdot, \cdot]^{\cdot}\right)$ is a Lie algebra and $\dot{\tau}: \dot{\mathcal{L}} \times \dot{\mathcal{L}} \rightarrow \operatorname{ker}(\pi)$ is a 2-cocycle.

In the following, we show that for $\lambda \in \Lambda, \dot{\mathcal{L}}^{\lambda}$ is a Lie subalgebra of $\dot{\mathcal{L}}$ and so $\dot{\mathcal{L}}$ is the direct union of the class $\left\{\dot{\mathcal{L}}^{\lambda} \mid \lambda \in \Lambda\right\}$ of its subalgebras.

Lemma 3.7. (i) (a) For $\lambda \in \Lambda, \dot{\mathcal{L}}^{\lambda}$ is a $\mathcal{G}^{\lambda}$-submodule of $\tilde{\mathcal{L}}$; in particular, $\dot{\mathcal{L}}$ is a $\mathcal{G}$-submodule of $\tilde{\mathcal{L}}$.

(b) $\left.\pi\right|_{\dot{\mathcal{L}}}$ is a Lie algebra isomorphism from $\left(\dot{\mathcal{L}},[\cdot, \cdot]^{\cdot}\right)$ to $(\mathcal{L},[\cdot, \cdot])$.

(c) For $\lambda \in \Lambda, \dot{\mathcal{L}}^{\lambda}$ is a Lie subalgebra of $\left(\dot{\mathcal{L}},[\cdot, \cdot]^{\cdot}\right)$ and $\left.\pi\right|_{\dot{\mathcal{L}}^{\lambda}}$ is a Lie algebra isomorphism from $\dot{\mathcal{L}}^{\lambda}$ to $\mathcal{L}^{\lambda}$.

(ii) (a) $\pi_{1}: \tilde{\mathcal{L}} \rightarrow \dot{\mathcal{L}}$ is a central extension of $\dot{\mathcal{L}}$ with corresponding 2-cocycle $\dot{\tau}$ satisfying $\dot{\tau}(\dot{\mathcal{G}}, \dot{\mathcal{L}})=\{0\}$.

(b) For $\lambda \in \Lambda,\left.\pi_{1}\right|_{\tilde{\mathcal{L}}^{\lambda}}: \tilde{\mathcal{L}}^{\lambda} \rightarrow \dot{\mathcal{L}}^{\lambda}$ is a central extension of $\dot{\mathcal{L}}^{\lambda}$ with corresponding 2 -cocycle $\left.\dot{\tau}\right|_{\dot{\mathcal{L}}^{\lambda} \times \dot{\mathcal{L}}^{\lambda}}$ satisfying $\dot{\tau}\left(\dot{\mathcal{G}}^{\lambda}, \dot{\mathcal{L}}^{\lambda}\right)=\{0\}$.

(iii) For $\lambda \in \Lambda$, there is a subalgebra $\dot{\mathcal{D}}_{\lambda}$ of $\dot{\mathcal{L}}^{\lambda}$ with $\pi\left(\dot{\mathcal{D}}_{\lambda}\right)=\mathcal{D}_{\lambda}$ such that

(1) $\dot{\mathcal{D}}_{0}=\dot{\mathcal{D}}$,

(2) $\dot{\mathcal{D}}_{\lambda}$ is a trivial $\dot{\mathcal{G}}^{\lambda}$-submodule of $\dot{\mathcal{L}}^{\lambda}$,

(3) $\dot{\mathcal{L}}^{\lambda}=\sum_{i \in \mathcal{I}} \dot{\mathcal{G}}_{i}^{\lambda} \oplus \sum_{j \in \mathcal{J}} \dot{\mathcal{S}}_{j}^{\lambda} \oplus \sum_{t \in \mathcal{T}} \dot{\mathcal{V}}_{t}^{\lambda} \oplus \dot{\mathcal{D}}_{\lambda}$

Proof. (i) (a) This follows easily from Lemma 3.6(i)(4).

(b) For each $x \in \tilde{\mathcal{L}}$, there are unique $\dot{\ell}_{x} \in \dot{\mathcal{L}}, \ell_{x} \in \mathcal{L}$ and $e_{x}, f_{x} \in \operatorname{ker}(\pi)$ such that $x=\dot{\ell}_{x}+e_{x}=\ell_{x}+f_{x}$. We have

$$
\begin{array}{ll}
\ell_{\dot{\ell}_{x}}=x, & f_{\dot{\ell}_{x}}=-e_{x}, \quad x \in \mathcal{L}, \\
\dot{\ell}_{\ell_{y}}=y, & e_{\ell_{y}}=-f_{y}, \quad y \in \dot{\mathcal{L}} .
\end{array}
$$

Using this, one can see that for $x, y \in \dot{\mathcal{L}}, \pi\left([x, y]^{\circ}\right)=\left[\ell_{x}, \ell_{y}\right]=[\pi(x), \pi(y)]$. This together with the fact that $\tilde{\mathcal{L}}=\mathcal{L} \oplus \operatorname{ker}(\pi)=\dot{\mathcal{L}} \oplus \operatorname{ker}(\pi)$ implies that $\pi$ restricted to $\dot{\mathcal{L}}$ is an isomorphism from $\dot{\mathcal{L}}$ onto $\mathcal{L}$.

(c) Suppose $\lambda \in \Lambda$ and $x, y \in \dot{\mathcal{L}}^{\lambda}$. Then

$$
[x, y]^{\cdot}=[x, y]^{\sim}-\dot{\tau}(x, y)=\left[\ell_{x}, \ell_{y}\right]+\tau\left(\ell_{x}, \ell_{y}\right)-\dot{\tau}(x, y) \in \mathcal{L}^{\lambda}+\operatorname{ker}(\pi)=\tilde{\mathcal{L}}^{\lambda} .
$$

Therefore we have $[x, y]^{\bullet} \in \dot{\mathcal{L}} \cap \tilde{\mathcal{L}}^{\lambda}=\dot{\mathcal{L}}^{\lambda}$. This shows that $\dot{\mathcal{L}}^{\lambda}$ is a subalgebra of $\dot{\mathcal{L}}$. Now as the restriction of $\pi$ to $\dot{\mathcal{L}}$ is a Lie algebra isomorphism from $\dot{\mathcal{L}}$ to $\mathcal{L},(3.15)$ 
shows that the restriction of $\pi$ to $\dot{\mathcal{L}}^{\lambda}$ is a Lie algebra isomorphism from $\dot{\mathcal{L}}^{\lambda}$ to $\mathcal{L}^{\lambda}$. This completes the proof.

(ii) (a) Since $\tilde{\mathcal{L}}=\dot{\mathcal{L}} \oplus \operatorname{ker}(\pi)$, it is immediate that $\left.\pi_{1}\right|_{\tilde{\mathcal{L}}}$ is a central extension of $\dot{\mathcal{L}}$. For the last assertion, suppose that $x \in \dot{\mathcal{G}}$ and $y \in \dot{\mathcal{L}}$. Then by Corollary 3.4(iii), $\pi(x) \in \mathcal{G}$ and so by part (i)(a), $[x, y]^{\sim}=[\pi(x), y]^{\sim} \in \dot{\mathcal{L}}$. This implies that $\dot{\tau}(x, y)=\pi_{2}\left([x, y]^{\sim}\right)=\pi_{2}\left([\pi(x), y]^{\sim}\right)=0$.

(b) Use the same argument as in (ii)(a).

(iii) Let $\lambda \in \Lambda$. Consider (3.4) and set $\dot{\mathcal{D}}_{\lambda}:=\dot{\mathcal{L}}^{\lambda} \cap \pi^{-1}\left(\mathcal{D}_{\lambda}\right)$. We first show that $\dot{\mathcal{D}}_{\lambda}$ is a subalgebra of $\dot{\mathcal{L}}^{\lambda}$. Suppose that $\dot{d}_{1}, \dot{d}_{2} \in \dot{\mathcal{D}}_{\lambda} \subseteq \dot{\mathcal{L}}^{\lambda}$. Since $\mathcal{D}_{\lambda}$ is a subalgebra of $\mathcal{L}^{\lambda}$, we have $\pi\left(\left[\dot{d}_{1}, \dot{d}_{2}\right]^{\circ}\right)=\pi\left(\left[\dot{d}_{1}, \dot{d}_{2}\right]^{\sim}\right)=\left[\pi\left(\dot{d}_{1}\right), \pi\left(\dot{d}_{2}\right)\right] \in \mathcal{D}_{\lambda}$. Therefore, $\left[\dot{d}_{1}, \dot{d}_{2}\right]^{*} \in \dot{\mathcal{L}}^{\lambda} \cap \pi^{-1}\left(\mathcal{D}_{\lambda}\right)=\dot{\mathcal{D}}_{\lambda}$. This means that $\dot{\mathcal{D}}_{\lambda}$ is a subalgebra of $\dot{\mathcal{L}}^{\lambda}$. It is easy to see that $\dot{\mathcal{D}}_{0}=\dot{\mathcal{D}}$. For $\left(\right.$ iii) $(2)$, we first recall that $\left.\pi\right|_{\tilde{\mathcal{L}}^{\lambda}}: \tilde{\mathcal{L}}^{\lambda} \rightarrow \mathcal{L}^{\lambda}$ is a central extension of $\mathcal{L}^{\lambda}$. We next note that $\pi\left(\dot{\mathcal{D}}_{\lambda}\right) \subseteq \mathcal{D}_{\lambda}$ and that by Proposition 3.3(ii) (c), we have $\pi\left(\dot{\mathcal{G}}^{\lambda}\right) \subseteq \mathcal{G}^{\lambda}$, so Lemma 3.1 implies that

$$
\left[\dot{\mathcal{G}}^{\lambda}, \dot{\mathcal{D}}_{\lambda}\right]^{\sim}=\left[\mathcal{G}^{\lambda}, \mathcal{D}_{\lambda}\right]^{\sim}=\{0\} .
$$

To show (iii)(3), we just need to prove that

$$
\dot{\mathcal{D}} \subseteq \sum_{i \in \mathcal{I}} \dot{\mathcal{G}}_{i}^{\lambda} \oplus \sum_{j \in \mathcal{J}} \dot{\mathcal{S}}_{j}^{\lambda} \oplus \sum_{t \in \mathcal{T}} \dot{\mathcal{V}}_{t}^{\lambda} \oplus \dot{\mathcal{D}}_{\lambda}
$$

Suppose that $\dot{d} \in \dot{\mathcal{D}}$. Since $\pi(\dot{\mathcal{D}}) \subseteq \mathcal{D}_{0}$, one can find $d_{0} \in \mathcal{D}_{0}$ and $r \in \operatorname{ker}(\pi)$ such that $\dot{d}=d_{0}+r$. But $\mathcal{D}_{0} \subseteq \mathcal{D}_{\lambda}+\sum_{j \in \mathcal{J}} \mathcal{S}_{j}^{\lambda}$ and $\sum_{j \in \mathcal{J}} \mathcal{S}_{j}^{\lambda} \subseteq \sum_{j \in \mathcal{J}} \dot{\mathcal{S}}_{j}^{\lambda}+\operatorname{ker}(\pi)$ (see (3.13)), so there are $d_{\lambda} \in \mathcal{D}_{\lambda}, \dot{s} \in \sum_{j \in \mathcal{J}} \dot{\mathcal{S}}_{j}^{\lambda}$ and $r^{\prime} \in \operatorname{ker}(\pi)$ such that $\dot{d}=d_{\lambda}+\dot{s}+r^{\prime}+r$. Thus $\dot{d}_{\lambda}:=d_{\lambda}+r^{\prime}+r=\dot{d}-\dot{s} \in \dot{\mathcal{L}}^{\lambda}$ and $\pi\left(\dot{d}_{\lambda}\right)=d_{\lambda} \in \mathcal{D}_{\lambda}$, so $\dot{d}_{\lambda} \in \dot{\mathcal{D}}_{\lambda}$. This shows that $\dot{d}=\dot{d}_{\lambda}+\dot{s} \in \dot{\mathcal{D}}_{\lambda} \oplus \sum_{j \in \mathcal{J}} \dot{\mathcal{S}}_{j}^{\lambda}$ and so we are done.

Now we are ready to turn to the main results of this section. For the convenience of readers, we summarize what we have done so far. We recall that $R$ is an irreducible locally finite root system of type $B C_{I}$ and

$$
\mathcal{L}=\sum_{i \in \mathcal{I}} \mathcal{G}_{i} \oplus \sum_{j \in \mathcal{J}} \mathcal{S}_{j} \oplus \sum_{t \in \mathcal{T}} \mathcal{V}_{t} \oplus \mathcal{D}_{0}=(\mathcal{G} \otimes \mathcal{A}) \oplus(\mathcal{S} \otimes \mathcal{B}) \oplus(\mathcal{V} \otimes \mathcal{C}) \oplus \mathcal{D}_{0}
$$

is an $R$-graded Lie algebra with grading pair $(\mathcal{G}, \mathcal{H})$. Also $\pi:(\tilde{\mathcal{L}},[\cdot, \cdot] \sim) \rightarrow(\mathcal{L},[\cdot, \cdot])$ is a central extension of $\mathcal{L}$ with corresponding 2-cocycle $\tau$. We saw that we can lift $\mathcal{L}$ (resp. $\mathcal{G}$ ) to a subspace $\dot{\mathcal{L}}($ resp. $\dot{\mathcal{G}}$ ) of $\tilde{\mathcal{L}}$ which can be identified with $\mathcal{L}$ (resp. $\mathcal{G}$ ) as a Lie algebra so that the corresponding 2-cocycle $\dot{\tau}$ satisfies

$$
\dot{\tau}(\dot{\mathcal{G}}, \dot{\mathcal{L}})=\{0\} .
$$

Also the Lie algebra $\dot{\mathcal{G}}$ is the direct union of a class $\left\{\dot{\mathcal{G}}^{\lambda} \mid \lambda \in \Lambda\right\}$ of its subalgebras and the Lie algebra $\dot{\mathcal{L}}$ is the direct union of a class $\left\{\dot{\mathcal{L}}^{\lambda} \mid \lambda \in \Lambda\right\}$ of its subalgebras. 
Moreover, there is a class

$$
\left\{\dot{\mathcal{G}}_{i}, \dot{\mathcal{S}}_{j}, \dot{\mathcal{V}}_{t}, \dot{\mathcal{G}}_{i}^{\lambda}, \dot{\mathcal{S}}_{j}^{\lambda}, \dot{\mathcal{V}}_{t}^{\lambda}, \dot{\mathcal{D}}_{\lambda} \mid \lambda \in \Lambda, i \in \mathcal{I}, j \in \mathcal{J}, t \in \mathcal{T}\right\}
$$

such that

$$
\dot{\mathcal{L}}=\sum_{i \in \mathcal{I}} \dot{\mathcal{G}}_{i} \oplus \sum_{j \in \mathcal{J}} \dot{\mathcal{S}}_{j} \oplus \sum_{t \in \mathcal{T}} \dot{\mathcal{V}}_{t} \oplus \dot{\mathcal{D}}_{0}
$$

and for $\lambda \in \Lambda$,

$$
\dot{\mathcal{L}}^{\lambda}=\sum_{i \in \mathcal{I}} \dot{\mathcal{G}}_{i}^{\lambda} \oplus \sum_{j \in \mathcal{J}} \dot{\mathcal{S}}_{j}^{\lambda} \oplus \sum_{t \in \mathcal{T}} \dot{\mathcal{V}}_{t}^{\lambda} \oplus \dot{\mathcal{D}}_{\lambda}
$$

is a decomposition of $\dot{\mathcal{L}}^{\lambda}$ into irreducible $\dot{\mathcal{G}}^{\lambda}$-submodules via the adjoint representation. Also for all $\lambda \in \Lambda$,

- $\dot{\mathcal{G}}_{0}=\dot{\mathcal{G}}$ and $\dot{\mathcal{G}}_{0}^{\lambda}=\dot{\mathcal{G}}^{\lambda}$,

- $\dot{\mathcal{G}}^{\lambda}$ is a subalgebra of $\tilde{\mathcal{L}}$,

- $\dot{\mathcal{D}}_{\lambda}$ is a subalgebra of the centralizer of $\dot{\mathcal{G}}^{\lambda}$ in $\dot{\mathcal{L}}^{\lambda}$,

- $\dot{\mathcal{D}}_{0} \subseteq\left[\mathcal{L}^{0}, \mathcal{L}^{0}\right]^{\sim}=\left[\dot{\mathcal{L}}^{0}, \dot{\mathcal{L}}^{0}\right]^{\sim}$,

- $\tilde{\mathcal{L}}=\dot{\mathcal{L}} \oplus \operatorname{ker}(\pi)=\sum_{i \in \mathcal{I}} \dot{\mathcal{G}}_{i} \oplus \sum_{j \in \mathcal{J}} \dot{\mathcal{S}}_{j} \oplus \sum_{t \in \mathcal{T}} \dot{\mathcal{V}}_{t} \oplus \dot{\mathcal{D}}_{0} \oplus \operatorname{ker}(\pi)$,

- $\tilde{\mathcal{L}}^{\lambda}=\dot{\mathcal{L}}^{\lambda} \oplus \operatorname{ker}(\pi)=\sum_{i \in \mathcal{I}} \dot{\mathcal{G}}_{i}^{\lambda} \oplus \sum_{j \in \mathcal{J}} \dot{\mathcal{S}}_{j}^{\lambda} \oplus \sum_{t \in \mathcal{T}} \dot{\mathcal{V}}_{t}^{\lambda} \oplus \dot{\mathcal{D}}_{0} \oplus \operatorname{ker}(\pi)$

$$
=\sum_{i \in \mathcal{I}} \dot{\mathcal{G}}_{i}^{\lambda} \oplus \sum_{j \in \mathcal{J}} \dot{\mathcal{S}}_{j}^{\lambda} \oplus \sum_{t \in \mathcal{T}} \dot{\mathcal{V}}_{t}^{\lambda} \oplus \dot{\mathcal{D}}_{\lambda} \oplus \operatorname{ker}(\pi) .
$$

So we may replace $\left(\mathcal{L}, \mathcal{G}, \tau, \mathcal{L}^{\lambda}, \mathcal{G}^{\lambda}, \mathcal{G}_{i}, \mathcal{S}_{j}, \mathcal{V}_{t}, \mathcal{G}_{i}^{\lambda}, \mathcal{S}_{j}^{\lambda}, \mathcal{V}_{t}^{\lambda}, \mathcal{D}_{\lambda}\right)$ (see (3.4), (3.5), (3.8) and $(3.9))$ with $\left(\dot{\mathcal{L}}, \dot{\mathcal{G}}, \dot{\tau}, \dot{\mathcal{L}}^{\lambda}, \dot{\mathcal{G}}^{\lambda}, \dot{\mathcal{G}}_{i}, \dot{\mathcal{S}}_{j}, \dot{\mathcal{V}}_{t}, \dot{\mathcal{G}}_{i}^{\lambda}, \dot{\mathcal{S}}_{j}^{\lambda}, \dot{\mathcal{V}}_{t}^{\lambda}, \dot{\mathcal{D}}_{\lambda}\right)$; in particular we may assume

$$
\begin{aligned}
\mathcal{L}^{\lambda} & =\left(\mathcal{G}^{\lambda} \otimes \mathcal{A}\right) \oplus\left(\mathcal{S}^{\lambda} \otimes \mathcal{B}\right) \oplus\left(\mathcal{V}^{\lambda} \otimes \mathcal{C}\right) \oplus \mathcal{D}_{\lambda} \\
& =\left(\mathcal{G}^{\lambda} \otimes \mathcal{A}\right) \oplus\left(\mathcal{S}^{\lambda} \otimes \mathcal{B}\right) \oplus\left(\mathcal{V}^{\lambda} \otimes \mathcal{C}\right) \oplus \mathcal{D}_{0}
\end{aligned}
$$

with

$$
\tau(\mathcal{G}, \mathcal{L})=\{0\} \quad \text { and } \quad \mathcal{D}_{0} \subseteq\left[\mathcal{L}^{0}, \mathcal{L}^{0}\right]^{\sim} .
$$

Now since $\left.\pi\right|_{\mathcal{L}^{\lambda} \oplus \operatorname{ker}(\pi)}$ is a central extension of $\mathcal{L}^{\lambda}$ with corresponding 2-cocycle $\tau$ satisfying $\tau\left(\mathcal{G}^{\lambda}, \mathcal{L}^{\lambda}\right)=\{0\}$, it follows from [3, Prop. 5.32] and [2, Thm. 3.7] that the summands $\mathcal{G}^{\lambda} \otimes \mathcal{A}, \mathcal{S}^{\lambda} \otimes \mathcal{B}, \mathcal{V}^{\lambda} \otimes \mathcal{C}$ and $\mathcal{D}_{\lambda}$ are orthogonal with respect to $\tau$ and that for $x, y \in \mathcal{G}^{\lambda}, a \in \mathcal{A}, s \in \mathcal{S}^{\lambda}, b \in \mathcal{B}, v \in \mathcal{V}^{\lambda}$ and $c \in \mathcal{C}$, we have

$$
\begin{aligned}
& {[x \otimes 1, y \otimes a]^{\sim}=[x \otimes 1, y \otimes a]+\tau(x \otimes 1, y \otimes a)=[x \otimes 1, y \otimes a]=[x, y] \otimes a,} \\
& {[x \otimes 1, s \otimes b]^{\sim}=[x \otimes 1, s \otimes b]+\tau(x \otimes 1, s \otimes b)=[x \otimes 1, s \otimes b]=[x, s] \otimes b,} \\
& {[x \otimes 1, v \otimes c]^{\sim}=[x \otimes 1, v \otimes c]+\tau(x \otimes 1, v \otimes c)=[x \otimes 1, v \otimes c]=x v \otimes c .}
\end{aligned}
$$


Together with the fact that $\mathcal{G}^{\lambda}, \mathcal{S}^{\lambda}$ and $\mathcal{V}^{\lambda}$ are irreducible finite-dimensional $\mathcal{G}$-modules, this implies that $\left(\mathcal{G}^{\lambda} \otimes \mathcal{A}\right) \oplus\left(\mathcal{S}^{\lambda} \otimes \mathcal{B}\right) \oplus\left(\mathcal{V}^{\lambda} \otimes \mathcal{C}\right) \subseteq\left[\mathcal{L}^{\lambda}, \mathcal{L}^{\lambda}\right]^{\sim}$. Therefore using (3.19), we conclude that for $\lambda \in \Lambda, \mathcal{L}^{\lambda} \subseteq\left[\mathcal{L}^{\lambda}, \mathcal{L}^{\lambda}\right]^{\sim}$; in particular, $\mathcal{L} \subseteq[\mathcal{L}, \mathcal{L}]^{\sim}$

Theorem 3.8. Suppose that $I$ is an infinite index set and $\ell$ is an integer greater than $3, R$ is an irreducible locally finite root system of type $B C_{I}$ and $\mathfrak{q}:=(\mathfrak{a}, *, \mathcal{C}, f)$ is a coordinate quadruple of type $B C$. Take $\mathfrak{b}:=\mathfrak{b}(\mathfrak{q})$ and suppose $\mathcal{K}$ is a subspace of $\mathfrak{U}(\mathfrak{b})$. Set $\langle\mathfrak{b}, \mathfrak{b}\rangle:=\{\mathfrak{b}, \mathfrak{b}\}_{\ell} / \mathcal{K}$, take $\mathcal{A}($ resp. $\mathcal{B})$ to be the set of $*$-fixed points (resp. *-skew-fixed points) of $\mathfrak{a}$ and consider the $R$-graded Lie algebra $\mathcal{L}:=(\mathcal{G} \otimes \mathcal{A}) \oplus$ $(\mathcal{S} \otimes \mathcal{B}) \oplus(\mathcal{V} \otimes \mathcal{C}) \oplus\langle\mathfrak{b}, \mathfrak{b}\rangle$. Use the same notation as in Theorem 2.8 and suppose that $\tau: \mathcal{L} \times \mathcal{L} \rightarrow E$ is a 2-cocycle. Consider the corresponding central extension $\tilde{\mathcal{L}}:=\mathcal{L} \oplus E$ as well as the canonical projection map $\pi: \tilde{\mathcal{L}} \rightarrow \mathcal{L}$. If $\tilde{\mathcal{L}}$ is perfect, then $\tilde{\mathcal{L}}$ is an $R$-graded Lie algebra with the same coordinate quadruple $(\mathfrak{a}, *, \mathcal{C}, f)$. Also there is a subspace $\mathcal{K}_{0} \subseteq \mathfrak{U}(\mathfrak{b})$ such that $\tilde{\mathcal{L}}$ can be identified with $(\mathcal{G} \otimes \mathcal{A}) \oplus$ $(\mathcal{S} \otimes \mathcal{B}) \oplus(\mathcal{V} \otimes \mathcal{C}) \oplus\left(\{\mathfrak{b}, \mathfrak{b}\}_{\ell} / \mathcal{K}_{0}\right)$ where setting $\left\langle\beta, \beta^{\prime}\right\rangle_{c}:=\left\{\beta, \beta^{\prime}\right\}_{\ell}+\mathcal{K}_{0}\left(\beta, \beta^{\prime} \in \mathfrak{b}\right)$, the Lie bracket on $\tilde{\mathcal{L}}$ is given by

$$
\begin{aligned}
& {\left[x \otimes a, y \otimes a^{\prime}\right]^{\sim}=[x, y] \otimes \frac{1}{2}\left(a \circ a^{\prime}\right)+(x \circ y) \otimes \frac{1}{2}\left[a, a^{\prime}\right]+\operatorname{tr}(x y)\left\langle a, a^{\prime}\right\rangle_{c},} \\
& {[x \otimes a, s \otimes b]^{\sim}=(x \circ s) \otimes \frac{1}{2}[a, b]+[x, s] \otimes \frac{1}{2}(a \circ b)=-[s \otimes b, x \otimes a]^{\sim},} \\
& {\left[s \otimes b, t \otimes b^{\prime}\right]^{\sim}=[s, t] \otimes \frac{1}{2}\left(b \circ b^{\prime}\right)+(s \circ t) \otimes \frac{1}{2}\left[b, b^{\prime}\right]+\operatorname{tr}(s t)\left\langle b, b^{\prime}\right\rangle_{c},} \\
& {[x \otimes a, u \otimes c]^{\sim}=x u \otimes a \cdot c=-[u \otimes c, x \otimes a]^{\sim},} \\
& {[s \otimes b, u \otimes c]^{\sim}=s u \otimes b \cdot c=-[u \otimes c, s \otimes b]^{\sim},} \\
& {\left[u \otimes c, v \otimes c^{\prime}\right]^{\sim}=(u \circ v) \otimes\left(c \diamond c^{\prime}\right)+[u, v] \otimes\left(c \circ c^{\prime}\right)+(u, v)\left\langle c, c^{\prime}\right\rangle_{c},} \\
& \left.\left[\left\langle\beta_{1}, \beta_{2}\right\rangle, x \otimes a\right]^{\sim}=\frac{-1}{4 \ell}\left(x \circ \mathfrak{I}_{0}\right) \otimes\left[a, \beta_{\beta_{1}, \beta_{2}}^{*}\right]+\left[x, \mathfrak{I}_{0}\right] \otimes a \circ \beta_{\beta_{1}, \beta_{2}}^{*}\right), \\
& {\left[\left\langle\beta_{1}, \beta_{2}\right\rangle, s \otimes b\right]^{\sim}=\frac{-1}{4 \ell}\left(\left[s, \mathfrak{I}_{0}\right] \otimes\left(b \circ \beta_{\beta_{1}, \beta_{2}}^{*}\right)+\left(s \circ \mathfrak{I}_{0}\right) \otimes\left[b, \beta_{\beta_{1}, \beta_{2}}^{*}\right]+2 \operatorname{tr}\left(s \mathfrak{I}_{0}\right)\left\langle b, \beta_{\beta_{1}, \beta_{2}}^{*}\right\rangle_{c}\right),} \\
& {\left[\left\langle\beta_{1}, \beta_{2}\right\rangle_{c}, v \otimes c\right]^{\sim}=\frac{1}{2 \ell} \Im_{0} v \otimes\left(\beta_{\beta_{1}, \beta_{2}}^{*} c\right)-\frac{1}{2} v \otimes\left(f\left(c, \beta_{2}^{*}\right) \cdot \beta_{1}^{*}+f\left(c, \beta_{1}^{*}\right) \cdot \beta_{2}^{*}\right)} \\
& {\left[\left\langle\beta_{1}, \beta_{2}\right\rangle_{c},\left\langle\beta_{1}^{\prime}, \beta_{2}^{\prime}\right\rangle_{c}\right]^{\sim}=\left\langle d_{\beta_{1}, \beta_{2}}^{\ell}\left(\beta_{1}^{\prime}\right), \beta_{2}^{\prime}\right\rangle_{c}+\left\langle\beta_{1}^{\prime}, d_{\beta_{1}, \beta_{2}}^{\ell}\left(\beta_{2}^{\prime}\right)\right\rangle_{c}}
\end{aligned}
$$

for $x, y \in \mathcal{G}, s, t \in \mathcal{S}, u, v \in \mathcal{V}, a, a^{\prime} \in \mathcal{A}, b, b^{\prime} \in \mathcal{B}, c, c^{\prime} \in \mathcal{C}, \beta_{1}, \beta_{2}, \beta_{1}^{\prime}, \beta_{2}^{\prime} \in \mathfrak{b}$. Moreover, under the above identification, $\pi: \tilde{\mathcal{L}} \rightarrow \mathcal{L}$ is given by $\pi(x)=x$ for $x \in(\mathcal{G} \otimes \mathcal{A}) \oplus(\mathcal{S} \otimes \mathcal{B}) \oplus(\mathcal{V} \otimes \mathcal{C})$ and $\pi\left(\left\langle\beta, \beta^{\prime}\right\rangle\right)=\left\langle\beta, \beta^{\prime}\right\rangle_{c}$ for $\beta, \beta^{\prime} \in \mathfrak{b}$.

Proof. As we have already seen, without loss of generality we may assume

$$
\tau(\mathcal{G}, \mathcal{L})=\{0\} \quad \text { and } \quad \mathcal{L}^{\lambda} \subseteq\left[\mathcal{L}^{\lambda}, \mathcal{L}^{\lambda}\right]^{\sim}, \quad \lambda \in \Lambda
$$

We know that $\tilde{\mathcal{L}}$ is an $R$-graded Lie algebra with grading pair $(\mathcal{G}, \mathcal{H})$ and weight space decomposition $\tilde{\mathcal{L}}=\bigoplus_{\alpha \in R} \tilde{\mathcal{L}}_{\alpha}$ where

$$
\tilde{\mathcal{L}}_{0}=\mathcal{L}_{0} \oplus \operatorname{ker}(\pi)=\mathcal{L}_{0} \oplus E \quad \text { and } \quad \tilde{\mathcal{L}}_{\alpha}=\mathcal{L}_{\alpha}, \quad \alpha \in R \backslash\{0\} .
$$


Suppose that $\left\{a_{i} \mid i \in \mathcal{I}\right\},\left\{b_{j} \mid j \in \mathcal{J}\right\}$ and $\left\{c_{t} \mid t \in \mathcal{T}\right\}$ are bases for $\mathcal{A}, \mathcal{B}$ and $\mathcal{C}$ respectively. We assume $0 \in \mathcal{I}$ with $a_{0}=1$ and identify $\mathcal{G} \otimes 1$ with $\mathcal{G}$. For $\lambda \in \Lambda$ and $i \in \mathcal{I}, j \in \mathcal{J}$ and $t \in \mathcal{T}$, we set

$$
\begin{array}{rlrl}
\mathcal{G}_{i}^{\lambda}:=\mathcal{G}^{\lambda} \otimes a_{i}, & & \mathcal{G}_{i}:=\mathcal{G} \otimes a_{i}, \\
\mathcal{S}_{j}^{\lambda}:=\mathcal{S}^{\lambda} \otimes b_{j}, & \mathcal{S}_{j}:=\mathcal{S} \otimes b_{j}, \\
\mathcal{V}_{t}^{\lambda}:=\mathcal{V}^{\lambda} \otimes c_{t}, & & \mathcal{V}_{t}:=\mathcal{V} \otimes c_{t} .
\end{array}
$$

Then $\mathcal{G}_{i}=\bigcup_{\lambda \in \Lambda} \mathcal{G}_{i}^{\lambda}, \mathcal{S}_{j}=\bigcup_{\lambda \in \Lambda} \mathcal{S}_{j}^{\lambda}$ and $\mathcal{V}_{t}=\bigcup_{\lambda \in \Lambda} \mathcal{V}_{t}^{\lambda}(i \in \mathcal{I}, j \in \mathcal{J}, t \in \mathcal{T})$ and for $\mathcal{D}:=\langle\mathfrak{b}, \mathfrak{b}\rangle$, we have $\mathcal{L}=\sum_{i \in \mathcal{I}} \mathcal{G}_{i} \oplus \sum_{j \in \mathcal{J}} \mathcal{S}_{j} \oplus \sum_{t \in \mathcal{T}} \mathcal{V}_{t} \oplus \mathcal{D}$. For $\lambda \in \Lambda$, set

$$
\begin{aligned}
& \mathcal{L}^{\lambda}:=\sum_{i \in \mathcal{I}} \mathcal{G}_{i}^{\lambda} \oplus \sum_{j \in \mathcal{J}} \mathcal{S}_{j}^{\lambda} \oplus \sum_{t \in \mathcal{T}} \mathcal{V}_{t}^{\lambda} \oplus \mathcal{D}, \\
& \tilde{\mathcal{L}}^{\lambda}:=\sum_{i \in \mathcal{I}} \mathcal{G}_{i}^{\lambda} \oplus \sum_{j \in \mathcal{J}} \mathcal{S}_{j}^{\lambda} \oplus \sum_{t \in \mathcal{T}} \mathcal{V}_{t}^{\lambda} \oplus \mathcal{D} \oplus \operatorname{ker}(\pi), \\
& \hat{\mathcal{L}}^{\lambda}:=\left[\tilde{\mathcal{L}}^{\lambda}, \tilde{\mathcal{L}}^{\lambda}\right]^{\sim}=\left[\mathcal{L}^{\lambda}, \mathcal{L}^{\lambda}\right]^{\sim} .
\end{aligned}
$$

The restriction of $\pi$ to $\tilde{\mathcal{L}}^{\lambda}$ is a central extension of $\mathcal{L}^{\lambda}$. Now $\pi_{\lambda}:=\left.\pi\right|_{\hat{\mathcal{L}}^{\lambda}}: \hat{\mathcal{L}}^{\lambda} \rightarrow \mathcal{L}^{\lambda}$ is a perfect central extension of $\mathcal{L}^{\lambda}$. Since $\mathcal{L}^{\lambda} \subseteq\left[\mathcal{L}^{\lambda}, \mathcal{L}^{\lambda}\right]^{\sim}$ for $\lambda \in \Lambda$, setting $\mathcal{Z}_{\lambda}:=\operatorname{ker}\left(\pi_{\lambda}\right)$, we have

$$
\hat{\mathcal{L}}^{\lambda}=\mathcal{L}^{\lambda} \oplus \mathcal{Z}_{\lambda}
$$

Also as $\tilde{\mathcal{L}}$ is perfect, we get

$$
\tilde{\mathcal{L}}=[\tilde{\mathcal{L}}, \tilde{\mathcal{L}}]^{\sim}=\left[\bigcup_{\lambda \in \Lambda} \tilde{\mathcal{L}}^{\lambda}, \bigcup_{\lambda \in \Lambda} \tilde{\mathcal{L}}^{\lambda}\right]^{\sim}=\bigcup_{\lambda \in \Lambda}\left[\tilde{\mathcal{L}}^{\lambda}, \tilde{\mathcal{L}}^{\lambda}\right]^{\sim}=\bigcup_{\lambda \in \Lambda} \hat{\mathcal{L}}^{\lambda},
$$

i.e., the Lie algebra $\tilde{\mathcal{L}}$ is the direct union of its subalgebras $\hat{\mathcal{L}}^{\lambda}$. We next note that

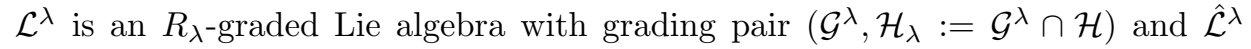
is a perfect central extension of $\mathcal{L}^{\lambda}$ with corresponding 2-cocycle $\tau_{\lambda}:=\left.\tau\right|_{\mathcal{L}^{\lambda} \times \mathcal{L}^{\lambda}}$ satisfying $\tau_{\lambda}\left(\mathcal{G}^{\lambda}, \mathcal{L}^{\lambda}\right)=\{0\}$. Therefore by Lemma 3.2,

$$
\hat{\mathcal{L}}^{\lambda}=\sum_{i \in \mathcal{I}} \mathcal{G}_{i}^{\lambda} \oplus \sum_{j \in \mathcal{J}} \mathcal{S}_{j}^{\lambda} \oplus \sum_{t \in \mathcal{T}} \mathcal{V}_{t}^{\lambda} \oplus \mathcal{D}_{\lambda} \oplus \mathcal{Z}_{\lambda}
$$

is an $R_{\lambda}$-graded Lie algebra and $\mathcal{D}_{\lambda} \oplus \mathcal{Z}_{\lambda}$ is a trivial $\mathcal{G}^{\lambda}$-submodule of $\hat{\mathcal{L}}^{\lambda}$. Now the structure of $\mathcal{G}_{i}^{0}, \mathcal{G}_{i}^{\lambda}, \mathcal{S}_{j}^{0}, \mathcal{S}_{j}^{\lambda}, \mathcal{V}_{t}^{0}$ and $\mathcal{V}_{t}^{\lambda}$ allows us to use the same argument as in [19, Rem. 3.9 and Thm. 4.1] to get

$$
\begin{aligned}
\hat{\mathcal{L}}^{\lambda} & =\sum_{i \in \mathcal{I}} \mathcal{G}_{i}^{\lambda} \oplus \sum_{j \in \mathcal{J}} \mathcal{S}_{j}^{\lambda} \oplus \sum_{t \in \mathcal{T}} \mathcal{V}_{t}^{\lambda} \oplus \mathcal{D}_{0} \oplus \mathcal{Z}_{0} \\
& =\left(\mathcal{G}^{\lambda} \otimes \mathcal{A}\right) \oplus\left(\mathcal{S}^{\lambda} \otimes \mathcal{B}\right) \oplus\left(\mathcal{V}^{\lambda} \otimes \mathcal{C}\right) \oplus \mathcal{D}_{0} \oplus \mathcal{Z}_{0}
\end{aligned}
$$


So

$$
\begin{aligned}
\tilde{\mathcal{L}}=\bigcup_{\lambda \in \Lambda} \hat{\mathcal{L}}^{\lambda} & =\sum_{i \in \mathcal{I}} \mathcal{G}_{i} \oplus \sum_{j \in \mathcal{J}} \mathcal{S}_{j} \oplus \sum_{t \in \mathcal{T}} \mathcal{V}_{t} \oplus \mathcal{D}_{0} \oplus \mathcal{Z}_{0} \\
& =(\mathcal{G} \otimes \mathcal{A}) \oplus(\mathcal{S} \otimes \mathcal{B}) \oplus(\mathcal{V} \otimes \mathcal{C}) \oplus \mathcal{D}_{0} \oplus \mathcal{Z}_{0}
\end{aligned}
$$

Now by $[19, \S 3]$ and Lemma $3.2, \mathcal{L}^{0}, \hat{\mathcal{L}}^{0}, \hat{\mathcal{L}}^{\lambda}$ and $\mathcal{L}^{\lambda}$ have the same coordinate quadruple, say $\mathfrak{q}$. Also there is a subspace $\mathcal{K}_{0} \subseteq \mathfrak{U}(\mathfrak{b})$, where $\mathfrak{b}:=\mathfrak{b}(\mathfrak{q})$, such that $\mathcal{D}_{0} \oplus \mathcal{Z}_{0}$ can be identified with $\{\mathfrak{b}, \mathfrak{b}\}_{\ell} / \mathcal{K}_{0}$. Now by [19, Prop. 3.10], we get (3.20) and so by Theorem 2.8, $\hat{\mathcal{L}}$ is an $R$-graded Lie algebra. Now for fixed $x, y \in \mathcal{G}$ with $\operatorname{tr}(x y) \neq 0$, and $a, a^{\prime} \in \mathcal{A}$, we have

$$
\begin{aligned}
{[x, y] \otimes \frac{1}{2}(a \circ} & \left.a^{\prime}\right)+(x \circ y) \otimes \frac{1}{2}\left[a, a^{\prime}\right]+\operatorname{tr}(x y)\left\langle a, a^{\prime}\right\rangle \\
& =\left[x \otimes a, y \otimes a^{\prime}\right]=\left[\pi(x \otimes a), \pi\left(y \otimes a^{\prime}\right)\right]=\pi\left(\left[x \otimes a, y \otimes a^{\prime}\right]^{\sim}\right) \\
& =\pi\left([x, y] \otimes \frac{1}{2}\left(a \circ a^{\prime}\right)+(x \circ y) \otimes \frac{1}{2}\left[a, a^{\prime}\right]+\operatorname{tr}(x y)\left\langle a, a^{\prime}\right\rangle_{c}\right) \\
& =[x, y] \otimes \frac{1}{2}\left(a \circ a^{\prime}\right)+(x \circ y) \otimes \frac{1}{2}\left[a, a^{\prime}\right]+\operatorname{tr}(x y) \pi\left(\left\langle a, a^{\prime}\right\rangle_{c}\right) .
\end{aligned}
$$

This implies $\pi\left(\left\langle a, a^{\prime}\right\rangle_{c}\right)=\left\langle a, a^{\prime}\right\rangle$. Similarly we can prove that $\pi\left(\left\langle b, b^{\prime}\right\rangle_{c}\right)=\left\langle b, b^{\prime}\right\rangle$ and $\pi\left(\left\langle c, c^{\prime}\right\rangle_{c}\right)=\left\langle c, c^{\prime}\right\rangle$ for $b, b^{\prime} \in \mathcal{B}$ and $c, c^{\prime} \in \mathcal{C}$.

Theorem 3.9. Suppose that $\ell$ is integer greater than 3 and $\mathfrak{q}:=(\mathfrak{a}, *, \mathcal{C}, f)$ is a coordinate quadruple of type $B C, \mathfrak{b}:=\mathfrak{b}(\mathfrak{q}), \mathcal{K} \subseteq \mathfrak{U}(\mathfrak{b})$ and $\langle\mathfrak{b}, \mathfrak{b}\rangle:=\{\mathfrak{b}, \mathfrak{b}\}_{\ell} / \mathcal{K}$. Consider the R-graded Lie algebra $\mathcal{L}:=\mathcal{L}(\mathfrak{q}, \mathcal{K})=(\mathcal{G} \otimes \mathcal{A}) \oplus(\mathcal{S} \otimes \mathcal{B}) \oplus(\mathcal{V} \otimes \mathcal{C}) \oplus\langle\mathfrak{b}, \mathfrak{b}\rangle$, where $\mathcal{A}$ is the set of $*$-fixed points of $\mathfrak{a}$ and $\mathcal{B}$ is the set of $*$-skew-fixed points of $\mathfrak{a}$. Set $\mathfrak{A}:=\mathcal{L}(\mathfrak{q},\{0\})=(\mathcal{G} \otimes \mathcal{A}) \oplus(\mathcal{S} \otimes \mathcal{B}) \oplus(\mathcal{V} \otimes \mathcal{C}) \oplus\{\mathfrak{b}, \mathfrak{b}\}_{\ell}$. Then

$$
\begin{aligned}
& \pi: \mathfrak{A} \rightarrow \mathcal{L}, \\
& x \mapsto x, \quad x \in(\mathcal{G} \otimes \mathcal{A}) \oplus(\mathcal{S} \otimes \mathcal{B}) \oplus(\mathcal{V} \otimes \mathcal{C}), \\
& \left\{\beta, \beta^{\prime}\right\}_{\ell} \mapsto\left\langle\beta, \beta^{\prime}\right\rangle:=\left\{\beta, \beta^{\prime}\right\}_{\ell}+\mathcal{K}, \quad \beta, \beta^{\prime} \in \mathfrak{b},
\end{aligned}
$$

is the universal central extension of $\mathcal{L}$, and $\operatorname{ker}(\pi)=\mathfrak{U}(\mathfrak{b})$.

Proof. By Theorem 2.8, $\mathfrak{A}$ is an $R$-graded Lie algebra. If $x \in \operatorname{ker}(\pi)$, then $x=$ $\sum_{i=1}^{n}\left\{\beta_{i}, \beta_{i}^{\prime}\right\}$ for some $\beta_{i}, \beta_{i}^{\prime} \in \mathfrak{b}, 1 \leq i \leq n$, such that $\sum_{i}\left\langle\beta_{i}, \beta_{i}^{\prime}\right\rangle=0$, so $x \in \mathcal{K}$. Therefore $\operatorname{ker}(\pi) \subseteq \mathcal{K} \subseteq \mathfrak{U}(\mathfrak{b})$. This together with (2.22) implies that $\operatorname{ker}(\pi) \subseteq$ $Z(\mathfrak{A})$. This means that $\pi$ is a central extension of $\mathcal{L}$. Now suppose that $\dot{\mathcal{L}}$ is a Lie algebra and $\dot{\varphi}: \dot{\mathcal{L}} \rightarrow \mathcal{L}$ is a central extension of $\mathcal{L}$. Let $\tilde{\mathcal{L}}$ be the derived algebra of $\dot{\mathcal{L}}$ and $\varphi:=\left.\dot{\varphi}\right|_{\tilde{\mathcal{L}}}$. Then $(\tilde{\mathcal{L}}, \varphi)$ is a perfect central extension of $\mathcal{L}$. By Theorem 3.8, we may assume that there is a subspace $\mathcal{K}_{0} \subseteq \mathfrak{U}(\mathfrak{b})$ such that $\tilde{\mathcal{L}}=$ $(\mathcal{G} \otimes \mathcal{A}) \oplus(\mathcal{S} \otimes \mathcal{B}) \oplus(\mathcal{V} \otimes \mathcal{C}) \oplus\langle\mathfrak{b}, \mathfrak{b}\rangle_{c}$ where $\langle\mathfrak{b}, \mathfrak{b}\rangle_{c}:=\{\mathfrak{b}, \mathfrak{b}\} / \mathcal{K}_{0}$ and that $\varphi: \tilde{\mathcal{L}} \rightarrow \mathcal{L}$ 
is given by

$$
\begin{aligned}
& \varphi(x)=x, \quad x \in(\mathcal{G} \otimes \mathcal{A}) \oplus(\mathcal{S} \otimes \mathcal{B}) \oplus(\mathcal{V} \otimes \mathcal{C}) \\
& \varphi\left(\left\langle\beta, \beta^{\prime}\right\rangle_{c}\right)=\left\langle\beta, \beta^{\prime}\right\rangle, \quad \beta, \beta^{\prime} \in \mathfrak{b} .
\end{aligned}
$$

Now

$$
\begin{aligned}
& \psi:(\mathcal{G} \otimes \mathcal{A}) \oplus(\mathcal{S} \otimes \mathcal{B}) \oplus(\mathcal{V} \otimes \mathcal{C}) \oplus\{\mathfrak{b}, \mathfrak{b}\} \rightarrow \hat{\mathcal{L}} \\
& \psi(x)=x, \quad x \in(\mathcal{G} \otimes \mathcal{A}) \oplus(\mathcal{S} \otimes \mathcal{B}) \oplus(\mathcal{V} \otimes \mathcal{C}), \quad \psi\left(\left\{\beta, \beta^{\prime}\right\}\right)=\left\langle\beta, \beta^{\prime}\right\rangle_{c}
\end{aligned}
$$

is a Lie algebra homomorphism satisfying $\varphi \circ \psi=\pi$. Altogether, $\pi$ is the universal central extension of $\mathcal{L}$. The last assertion immediately follows from (2.22).

Considering $[19$, Thms. 4.3, 4.4] and using the same argument as above, one gets the following theorem:

Theorem 3.10. Suppose that $\ell$ is a positive integer greater than 5 and $\mathfrak{q}:=$ $(\mathfrak{a}, *, \mathcal{C}, f)$ is a coordinate quadruple of type $X \neq B C, \mathfrak{b}:=\mathfrak{b}(\mathfrak{q}), \mathcal{K} \subseteq \mathfrak{U}(\mathfrak{b})$ and $\mathcal{L}:=\mathcal{L}(\mathfrak{q}, \mathcal{K})=(\mathcal{G} \otimes \mathcal{A}) \oplus(\mathcal{S} \otimes \mathcal{B}) \oplus\langle\mathfrak{b}, \mathfrak{b}\rangle$, the corresponding R-graded Lie algebra, where $\langle\mathfrak{b}, \mathfrak{b}\rangle:=\{\mathfrak{b}, \mathfrak{b}\}_{\ell} / \mathcal{K}$ and $\mathcal{A}$ (resp. $\left.\mathcal{B}\right)$ is the set of $*$-fixed (resp. *-skew-fixed) points of $\mathfrak{a}$. Then any perfect central extension of $\mathcal{L}$ is an $R$-graded Lie algebra with the same coordinate quadruple $\mathfrak{q}$. Moreover, for $\mathfrak{A}:=\mathcal{L}(\mathfrak{q},\{0\})=$ $(\mathcal{G} \otimes \mathcal{A}) \oplus(\mathcal{S} \otimes \mathcal{B}) \oplus\{\mathfrak{b}, \mathfrak{b}\}_{\ell}$

$$
\begin{aligned}
& \pi: \mathfrak{A} \rightarrow \mathcal{L}, \\
& x \mapsto x, \quad x \in(\mathcal{G} \otimes \mathcal{A}) \oplus(\mathcal{S} \otimes \mathcal{B}), \\
& \left\{\beta, \beta^{\prime}\right\}_{\ell} \mapsto\left\{\beta, \beta^{\prime}\right\}_{\ell}+\mathcal{K}, \quad \beta, \beta^{\prime} \in \mathfrak{b},
\end{aligned}
$$

is the universal central extension of $\mathcal{L}$, and $\operatorname{ker}(\pi)=\mathfrak{U}(\mathfrak{b})$.

\section{Acknowledgements}

This research was in part supported by a grant from IPM (No. 91170415) and partially carried out in IPM-Isfahan Branch.

\section{References}

[1] B. N. Allison, S. Azam, S. Berman, Y. Gao and A. Pianzola, Extended affine Lie algebras and their root systems, Mem. Amer. Math. Soc. 126 (1997), no. 603, x+122 pp. Zbl 0879.17012 MR 1376741

[2] B. N. Allison, G. Benkart and Y. Gao, Central extensions of Lie algebras graded by finite root systems, Math. Ann. 316 (2000), 499-527. Zbl 0989.17004 MR 1752782

[3] Lie algebras graded by the root systems $B C_{r}, r \geq 2$, Mem. Amer. Math. Soc. 158 (2002), no. 751, x+158 pp. Zbl 0998.17031 MR 1902499

[4] B. N. Allison and Y. Gao, The root system and the core of an extended affine Lie algebra, Selecta Math. (N.S.) 7 (2001), 149-212. Z Zbl 1058.17012 MR 1860013 
[5] S. Azam, H. Yamane and M. Yousofzadeh, A finite presentation of universal coverings of Lie tori, Publ. RIMS Kyoto Univ. 46 (2010), 507-548. Zbl 1204.17018 MR 2760736

[6] G. Benkart and O. Smirnov, Lie algebras graded by the root system $B C_{1}$, J. Lie Theory $\mathbf{1 3}$ (2003), 91-132. Zbl 1015.17028 MR 1958577

[7] G. Benkart and E. Zelmanov, Lie algebras graded by finite root systems and intersection matrix algebras, Invent. Math. 126 (1996), 1-45. Zbl 0871.17024 MR 1408554

[8] S. Berman and R. Moody, Lie algebras graded by finite root systems and the intersection matrix algebras of Slodowy, Invent. Math. 108 (1992), 323-347. Zbl 0778.17018 MR 1161095

[9] O. Loos and E. Neher, Locally finite root systems, Mem. Amer. Math. Soc. 171 (2004), no. 811, x+214 pp. Zbl 1195.17007 MR 2073220

[10] E. Neher, Lie algebras graded by 3-graded root systems and Jordan pairs covered by grids, Amer. J. Math. 118 (1996), 439-491. Zbl 0857.17019 MR 1385288

[11] _ Extended affine Lie algebras and other generalizations of affine Lie algebrasa survey, in Developments and trends in infinite-dimensional Lie theory, Progr. Math. 288, Birkhäuser Boston, 2011, 53-126. Zbl 1261.17023 MR 2743761

[12] E. Neher and J. Sun, Universal central extensions of direct limits of Lie superalgebras, J. Algebra 368 (2012), 169-181. Zbl 06154884 MR 2955226

[13] G. B. Seligman, Rational methods in Lie algebras, Lecture Notes in Pure Appl. Math. 17, Dekker, New York, 1976. Zbl 0334.17002 MR 0427394

[14] N. Stumme, The structure of locally finite split simple Lie algebras, J. Algebra 220 (1990), 664-693. Zbl 1027.17019 MR 1717365

[15] A. Welte, Central extensions of graded Lie algebras, Ph.D. thesis, Univ. Ottawa, 2009, 140 pp. MR 2753169

[16] Y. Yoshii, Root systems extended by an abelian group, their Lie algebras and the classification of Lie algebras of type B, J. Lie Theory 14 (2004), 371-394. Z Zbl 1087.17008 MR 2066861

[17] _ Lie tori-a simple characterization of extended affine Lie algebras, Publ. RIMS Kyoto Univ. 42 (2006), 739-762. Zbl 1148.17017 MR 2266995

[18] M. Yousofzadeh, A presentation of Lie tori of type $B_{\ell}$, Publ. RIMS Kyoto Univ. 44 (2008), 1-44. Zbl 1214.17010 MR 2405865

[19] _ Structure of root graded Lie algebras, J. Lie Theory 22 (2012), 397-435. Zbl 06049458 MR 2976925 